\title{
Tournament selection mechanism based random vector selection in differential evolution algorithm
}

\author{
Qamar Abbas ${ }^{1, *}$, Jamil Ahmad ${ }^{2}$, Hajira Jabeen ${ }^{1}$ \\ ${ }^{1}$ Computer Department, Iqra University, Islamabad,44000, Pakistan \\ ${ }^{2}$ Computer Department, Abasyn University, Islamabad,44000, Pakistan
}

\section{A R T I C L E I N F O}

\section{Article history:}

Received 14 August 2016

Received in revised form

18 May 2017

Accepted 7 June 2017

\section{Keywords:}

Differential evolution

Mutation

Crossover

Tournament

Random vector

\begin{abstract}
A B S T R A C T
Differential Evolution (DE) is a simple, powerful and easy to use global optimization algorithm. Trial vector generation mechanism influences the performance of DE algorithm significantly. This research work explores that whether random vector selection in trial vector generation have any role in improving the performance of DE algorithm. A novel tournament selection framework in DE algorithm is proposed to enhance its convergence speed. The novel TSRVDE framework employs tournament selection criteria focuses on the selection of random vector in DE trial vector. We can get rid of worst performing individual selection by TSRVDE that will be helpful to enhance the searching capability of DE algorithm. TSRVDE advancement is applied on the set of frequently used DE variants. To evaluate the performance of TSRVDE a test suit of comprehensive set of well-known multidimensional global optimizations problems is used. The acceleration of TSRVDE can be observed in the experimental results.
\end{abstract}

(C) 2017 The Authors. Published by IASE. This is an open access article under the CC BY-NC-ND license (http://creativecommons.org/licenses/by-nc-nd/4.0/).

\section{Introduction}

Differential evolution (DE), proposed by Storn and Price (1997) is a stochastic population based evolutionary algorithm. The advantage of DE over other evolutionary algorithms is that it is simple, easy to use, and speedy as well as increases the chance of searching global minima for function optimization (Brest et al., 2006; Price et al., 2005). DE has been successfully used in various real life fields like electrical power systems (Chiou et al., 2005), microwave engineering (Guo and Li, 2009), robotics (Liu et al., 2006), Bioinformatics (Garlapati and Banerji, 2010), chemical engineering (Xue-Feng et al. 2006), pattern recognition (Kang et al., 2014), artificial neural networks (Dos Santos Coelho and Guerra, 2008), signal processing (Luitel and Venayagamoorthy, 2008) etc. DE algorithm consists of populations of potential solutions that initialized randomly with in specified search space of ndimensional. All population members have equal chance to be selected as parent in DE algorithm. Potential solutions locate the optima by searching the whole search space. At each iteration of DE

\footnotetext{
* Corresponding Author.

Email Address: qamar.bhk@gmail.com (Q.Abbas)

https://doi.org/10.21833/ijaas.2017.07.022

2313-626X/C) 2017 The Authors. Published by IASE.

This is an open access article under the CC BY-NC-ND license

(http://creativecommons.org/licenses/by-nc-nd/4.0/)
}

algorithm trial vector of each population member is generated by summing the amplified weighted difference of two vectors to another vector (De Oliveira and Saramago, 2008). DE algorithm has shown to have better performance than Particle Swarm Optimization and Genetic Algorithm for numerical benchmark optimization (Das et al., 2008a and 2008b; Xu and Li, 2007). DE algorithm has few parameters like mutation probability ' $\mathrm{F}$ ', Crossover 'CR' and Population 'NP'. Mutation strategies in DE algorithms are formed by the linear combination of current population members. Mutant vectors in DE are created by using target vector and trial vector. Throughout this paper $\mathrm{V}_{\mathrm{i}}$ denotes the mutant vector, $X_{i}$ represents the target vector and $U_{i}$ denotes the trial vector. Throughout this paper $X_{i}$ denotes the target vector (or current vector), $U_{i}$ represents the trial vector and $\mathrm{V}_{\mathrm{i}}$ as a mutant vector. Various vectors like random vectors, best vector and current vector's combination is used to for form a trial vector in DE algorithm. The performance of DE algorithm is sensitive to crossover scheme, mutation strategy, mutation probability control parameter and crossover rate control parameter (Storn and Price, 1997; Das et al., 2009). Various vectors like best vector, current vector and random vector(s) are used to form mutation strategies in DE algorithms. Throughout the following analysis $x_{g}^{r_{k}}$ states the $\mathrm{g}^{\text {th }}$ generation $r_{k}^{\text {th }}$ random vector, the $\mathrm{g}^{\text {th }}$ generation donor vector component is used as $v_{g}^{i}$ to refer its $\mathrm{i}^{\text {th }}$ 
component, $x_{g}^{\text {best }}$ states the best vector at $\mathrm{g}^{\text {th }}$ generation and $g^{\text {th }}$ generation current vector is refer as $x_{g}^{i}$. The most commonly used DE variants given in
Table 1 have been used by many researchers in their research work (Gosh et al., 2011; Islam et al., 2012).

Table 1: Commonly used DE mutation strategies

\begin{tabular}{ccl}
\hline S. No & Name & \multicolumn{1}{c}{ Equation } \\
\hline $\mathrm{DE}_{1}$ & "DE"/rand/1/bin & $v_{g}^{i}=x_{g}^{r 1}+F\left(x_{g}^{r 2}-x_{g}^{r 3}\right)$ \\
$\mathrm{DE}_{2}$ & "DE"/best/1/bin & $v_{g}^{i}=x_{g}^{\text {best }}+F\left(x_{g}^{r 1}-x_{g}^{r 2}\right)$ \\
$\mathrm{DE}_{3}$ & "DE"/rand/2/bin & $v_{g}^{i}=x_{g}^{r 1}+F\left(x_{g}^{r 2}-x_{g}^{r 3}\right)+F\left(x_{g}^{r 4}-x_{g}^{r 5}\right)$ \\
$\mathrm{DE}_{4}$ & "DE"/best/2/bin & $v_{g}^{i}=x_{g}^{\text {best }}+F\left(x_{g}^{r 1}-x_{g}^{r 2}\right)+F\left(x_{g}^{r 3}-x_{g}^{r 4}\right)$ \\
$\mathrm{DE}_{5}$ & “DE"/rand to best/1/bin & $v_{g}^{i}=x_{g}^{i}+F\left(x_{g}^{\text {best }}-x_{g}^{i}\right)+F\left(x_{g}^{r 1}-x_{g}^{r 2}\right)$ \\
$\mathrm{DE}_{6}$ & "DE"/rand/1/exp & $v_{g}^{i}=x_{g}^{r 1}+F\left(x_{g}^{r 2}-x_{g}^{r 3}\right)$ \\
$\mathrm{DE}_{7}$ & "DE"/best/1/exp & $v_{g}^{i}=x_{g}^{\text {best }}+F\left(x_{g}^{r 1}-x_{g}^{r 2}\right)$ \\
$\mathrm{DE}_{8}$ & "DE"/rand/2/exp & $v_{g}^{i}=x_{g}^{r 1}+F\left(x_{g}^{r 2}-x_{g}^{r 3}\right)+F\left(x_{g}^{r 4}-x_{g}^{r 5}\right)$ \\
$\mathrm{DE}_{9}$ & "DE"/best/2/exp & $v_{g}^{i}=x_{g}^{\text {best }}+F\left(x_{g}^{r 1}-x_{g}^{r 2}\right)+F\left(x_{g}^{r 3}-x_{g}^{r 4}\right)$ \\
$\mathrm{DE}_{10}$ & "DE"/rand to best/1/exp & $v_{g}^{i}=x_{g}^{i}+F\left(x_{g}^{\text {best }}-x_{g}^{i}\right)+F\left(x_{g}^{r 1}-x_{g}^{r 2}\right)$ \\
\hline
\end{tabular}

Epitropakis et al. (2011) have incorporated proximity based mutation to enhance the performance of DE algorithm. The proximity based mutation utilizes neighbors instead of random indices $r_{1}, r_{2}, r_{3}$ to create a parent in DE algorithm. In have generated proximity based mutation strategies corresponding to each mutation strategy of $\mathrm{DE}$ algorithm that replaces each random with a selected neighbour. Cai and wang (2013) have introduced ranking based mutation operators in $\mathrm{DE}$ algorithm. In this research each parent in DE is selected based on the ranking since good performing population member produces good quality offspring members. Li et al. (2013) have introduced modified DE algorithm in their research work. They have used best of random mutation strategy and randomized local search in modified DE algorithm. Best of random mutation selected best performing individual from three selected individuals and then uses this best individual as a based vector. The local search does not use any analytical gradient rather it uses fraction of difference of maximum and minimum of current dimension using a random variable generated using Gaussian distribution having zero means and 1 standard deviation. Liang et al. (2011) have proposed Chaotic Differential Evolution (CDE) algorithm in their research work. CDE is based on cat map that utilizes chaotic sequence in searching optima. The Chaotic sequence is obtained by cat map helps DE algorithm is escaping from local optima. Ali et al. (2011) have introduced the concept of mixed mutation strategies in DE algorithm (MSDE). In MSDE conventional mutation strategies and interpolation based mutation strategies are integrated by using the concept of game theory. Research result shows the significant performance of MSDE. Piotrowski and Napiorkowski (2010) have proposed grouping based DE (GDE) algorithm in their research work. They have divided the population into four groups where three groups communicate rarely and fourth one communicate with all groups to obtain to keep it updated from the current population knowledge during the search process. Research results shows that GDE has encouraging results. Wang et al., (2010) have incorporated enhanced neighborhood search mechanism in DE algorithm (DENS) in their research work. The proposed algorithm creates two trial individuals for each population member by using global and local search technique and replaces the current individual by the fitter one. Halder et al. (2013) have introduced the cluster based differential evolution in their research. The proposed algorithm uses the concept of multi-population that divided the population into mutually exclusive clusters where number of cluster is an adaptive parameter. K-mean clustering algorithm is used on main population to form clusters. Then DE algorithm is used to evolve each cluster and knowledge of each cluster is shared periodically. Mininno et al. (2011) have introduced statistic description based Compact Differential Evolution (cDE) in their research work. This algorithm processes the statistic description of population that makes it capable to perform efficiently. Gong et al., (2011) have proposed strategy adaption mechanism (SaM) in DE algorithm to choose more suitable adaption strategy to solve any problem. In proposed mechanism different mutation strategies can use different parameter adaption scheme. Wang et al. (2013) have introduced Gaussian Bare-Bones DE (GBDE) algorithm and its modified version (MGBDE) in their research work. The GBDE algorithm uses Gaussian sampling in its mutation strategies. Due to randomness Gaussian sampling, GBDE has slow convergence speed. The convergence speed can be improved in its modified version that randomly assign a mutation strategy during population and that strategy does not changes during evolutionary process. Choi et al. (2013) have introduced parameter adaptive DE algorithm based on Cauchy distribution in their research work. The adaption of control parameter is decided in the selection operation that whether the population member has suitable control parameters or they should be changes using the proposed mechanism. This parameter adaption is done at each generation of the search process because if a population individual does not have good parameter values the created offspring might fail to evolve. Elsayed et al. (2013) have introduced improved self-adaptive DE algorithm in their research work. The proposed version uses mixture of four different mutation operators and two crossover operators because 
different operator's performance can vary at various stages of search process. Cai and Wang (2013) have introduced neighborhood and direction information based DE algorithm in their research work. This proposed version is composed of two operators for selection and mutation. Selection scheme is guided by the neighbour's that is done by the probability selection method by selecting base vector using tournament selection criteria. To generate offspring the direction of neighbours is incorporated in mutation strategy. Zhong et al. (2013) have used to concept of dual population in DE algorithm. Both the population shares their knowledge during the evolution process. This enhancement in DE improves the diversity and convergence speed of DE algorithm. Secmen and Tasgetiren (2013) have ensemble various DE algorithms in their research work. They have ensembles "DE/rand/1/bin", "DE/best/1/bin" and "DE/rand-to-best/1/bin" in optimization of lossless dielectric spheres. Slowik (2011) has introduced multiple trial vector DE versions in artificial neural network training. Research result shows that the proposed algorithm have better performance than singe trial vector DE version, Back propagation algorithm and LevenbergMarquardt method. Various state-of-the-art DE algorithms versions like FADE (Liu and Lampinen, 2005), jDE (Brest et al., 2006), ADE (Zaharie, 2003), JADE (Zhang and Sanderson, 2009), SaDE (Qin et al., 2009), DEGL (Das et al., 2009), CoDE (Wang et al., 2011) ,EPSDE (Mallipeddi et al., 2011), MDE_pBX (Islam et al., 2012), etc. are based on adaption / selection of parameters as well as adaption / selection of mutation strategies mechanisms.

\section{DE algorithm}

$\mathrm{DE}$ is a population based algorithm that consists of NP population members $x_{G}^{i}, i=1,2,3, \ldots \ldots, N P$ at $\mathrm{G}^{\text {th }}$ generation. One population member $x_{G}^{i}$ contains $\mathrm{D}$ dimensions that are constrained by the specified feasible region $\left[x_{\min , j}, x_{\max , j}\right], j=1,2, \ldots, D . \quad$ The population individuals are uniformly distributed in the search space in search for global optima. DE algorithm creates donor vector by mutation operation, trial vector by using crossover operation and then in selection operation it is determined whether trial vector or target vector will move survive into the new population.

\subsection{Mutation}

A donor vector $v_{i, G+1}$ is created for each target population members $x_{G}^{i}, i=1,2,3, \ldots, N P$ at current generation $G$ in $D E$ mutation operation. The donor vector in conventional $\mathrm{DE}$ algorithm is created by using the Eq. 1.

$V_{i, G+1}=x_{r 1, G}+F\left(x_{r 2, G}-x_{r 3, G}\right)$

With indexed $r_{1}, r_{2}$ and $r_{3}$ selected randomly from $[1, N P]$, these indexes are different from each other as well as running index $\mathrm{i}$ and $F$ is used to control the amplification of difference vector.

\subsection{Crossover}

To form a trial vector $u_{i, G}=$ $\left(u_{i, 1, G}, u_{i, 2, G}, \ldots \ldots \ldots, u_{i, D, G}\right)$ dimensions of mutated vector $v_{i, G+1}$ and target vector $x_{G}^{i}$ are swapped. The two most commonly used crossover schemes in DE algorithm are Binomial and exponential (Storn and Price, 1997; Mezura-Montes et al., 2006; Ali et al., 2009a, 2009b). Trial vector $u_{i, G}=$ $\left(u_{i, 1, G}, u_{i, 2, G}, \ldots \ldots \ldots, u_{i, D, G}\right)$ using the Binomial crossover scheme can be created by using Eq. 2:

$u_{i, G}= \begin{cases}v_{i, j, G} \text { if }\left(\operatorname{rand} j(0,1) \leq C R \text { or } j=j_{\text {rand }}\right) \\ x_{i, j} \text { otherwise }\end{cases}$

With a random number $j_{\text {rand }}$ from [1, D], mutant vector $v_{i, j, G}$; a small random number between 0 and $1, \operatorname{rand} j(0,1)$, CR parameter from $(0,1]$. The other crossover scheme is Exponential crossover scheme that uses Eq. 3 to generate trial vector $u_{i, j}$

$u_{i, G}=$

$\left\{\begin{array}{l}v_{i, j, G} \text { for } j=<l>_{D}+<l+1>_{D}+\cdots+<l+L-1>_{D} \\ x_{i, j} \quad \text { otherwise }\end{array}\right.$

where $\left\langle>_{D}\right.$ is modulo function with modulus $\mathrm{D} ; i=$ $1,2,3, \ldots . N P$ and $j=1,2,3, \ldots . D . \quad \mathrm{L}$ is a random number that is generated from $[1, D]$ also $l$ denotes a starting index $l$ that is selected randomly from $[1, D]$.

\subsection{Selection}

In selection it is determined that whether trial vector $u_{i, G}$ or target vector $x_{i, G}$ can survive to be used in next generation of DE algorithm. The following selection scheme is used (Eq. 4)

$x_{i, G+1}= \begin{cases}u_{i, G+1} & \text { if }\left(f\left(u_{i, G+1}\right)<f\left(x_{i, G}\right)\right) \\ x_{i, G} \quad \text { otherwise }\end{cases}$

where $f\left(u_{i, G+1}\right)$ refers to the fitness of trial vector and $f\left(x_{i, G}\right)$ refers to the fitness of target vector.

\section{Tournament selection mechanism based random vector selection in DE (TSRVDE) algorithm}

The proposed TSRVDE is based on the tournament selection mechanism in selecting random vector to generate child population members. Trial vector generation in DE utilizes current, best and random vector (s). A tournament of size $t$ is used to select the parents in generating trial vector of TSRVDE variants. For each random vector used in the equation of the variants, a tsrvde vector will be generated by using the criteria of tournament selection. By selecting random in this manner, selection of comparatively weak population 
members have fewer chance to be selected as a parent however the value of $\mathrm{k}$ is kept very small $(\mathrm{t}=3)$ to maintain the diversity in the child population that can be beneficial in escaping from local optima. Random selection of population members to be part of the tournament makes them almost equally likely to be selected as a parent. Healthy population members will generate healthy child population that makes it possible to enhance the convergence speed of TSRVDE. Since size of tournament is small and individuals of tournament are randomly selected which ensures randomness in TSRVDE. TRRVDE performance is compared with DE in the experimental results section of this paper. Commonly used DE mutation strategies are given in section-I of this paper, the corresponding TSRVDE mutation strategies are given in Table 2. Each trsv vector in the equations of Table 2 are generated by proposed TSRVDE mechanism (Fig. 1).

Table 2: TSRVDE versions of commonly used DE variants

\begin{tabular}{|c|c|c|}
\hline S. No & Name & Equation \\
\hline$T S R V D E_{1}$ & TSRVDE / rand/1/bin & $v_{g}^{i}=x_{g}^{t s r v 1}+F\left(x_{g}^{t s r v 2}-x_{g}^{t s r v 3}\right)$ \\
\hline TSRVDE $E_{2}$ & TSRVDE /best/1/bin & $v_{g}^{i}=x_{g}^{\text {best }}+F\left(x_{g}^{\text {tsrv1 }}-x_{g}^{t s r v 2}\right)$ \\
\hline$T_{S R V D E}$ & TSRVDE $/ \mathrm{rand} / 2 / \mathrm{bin}$ & $v_{g}^{i}=x_{g}^{t s r v 1}+F\left(x_{g}^{t s r v 2}-x_{g}^{t s r v 3}\right)+F\left(x_{g}^{t s r v 4}-x_{g}^{t s r v 5}\right)$ \\
\hline$T_{S R V D E}$ & TSRVDE /best/2/bin & $v_{g}^{i}=x_{g}^{\text {best }}+F\left(x_{g}^{t s r v 1}-x_{g}^{t s r v 2}\right)+F\left(x_{g}^{t s r v 3}-x_{g}^{t s r v 4}\right)$ \\
\hline$T S R V D E_{5}$ & TSRVDE /rand to best/1/bin & $v_{g}^{i}=x_{g}^{i}+F\left(x_{g}^{\text {best }}-x_{g}^{i}\right)+F\left(x_{g}^{t s r v 1}-x_{g}^{t s r v 2}\right)$ \\
\hline TSRVDE 6 & TSRVDE /rand/1/exp & $v_{g}^{i}=x_{g}^{t s r v 1}+F\left(x_{g}^{t s r v 2}-x_{g}^{t s r v 3}\right)$ \\
\hline $\operatorname{TSRVDE}_{7}$ & TSRVDE / best/1/ exp & $v_{g}^{i}=x_{g}^{\text {best }}+F\left(x_{g}^{t s r v 1}-x_{g}^{t s r v 2}\right)$ \\
\hline$T S R V D E_{8}$ & TSRVDE $/ \mathrm{rand} / 2 / \exp$ & $v_{g}^{i}=x_{g}^{t s r v 1}+F\left(x_{g}^{t s r v 2}-x_{g}^{t s r v 3}\right)+F\left(x_{g}^{t s r v 4}-x_{g}^{t s r v 5}\right)$ \\
\hline TSRVDE 9 & TSRVDE /best/2/ exp & $v_{g}^{i}=x_{g}^{\text {best }}+F\left(x_{g}^{t r v 1}-x_{g}^{t r r v 2}\right)+F\left(x_{g}^{t r r v 3}-x_{g}^{t s r v 4}\right)$ \\
\hline$T S R V D E_{10}$ & TSRVDE /rand to best/1/ exp & $v_{g}^{i}=x_{g}^{i}+F\left(x_{g}^{\text {best }}-x_{g}^{i}\right)+F\left(x_{g}^{t s r v 1}-x_{g}^{t s r v 2}\right)$ \\
\hline
\end{tabular}

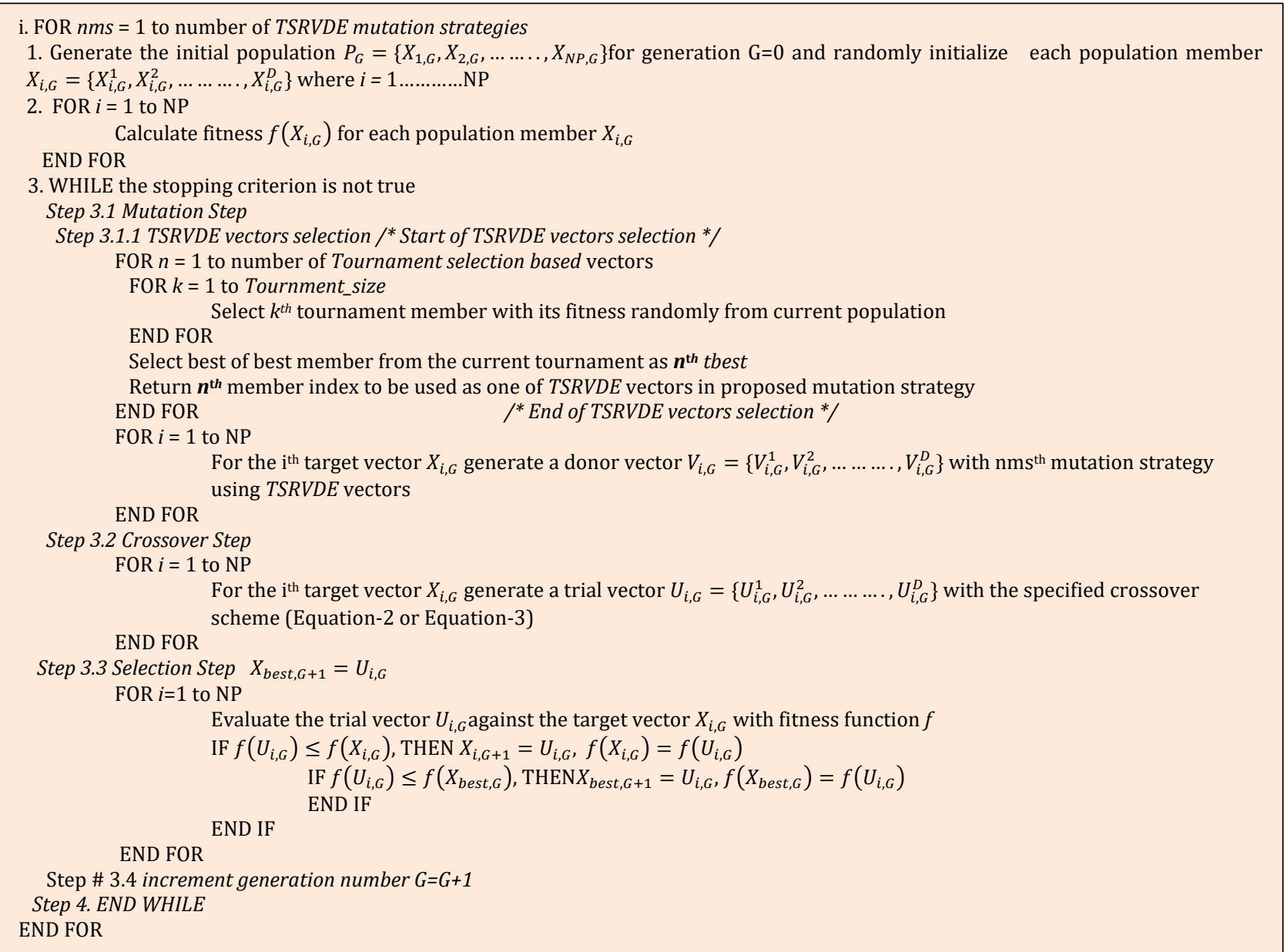

Fig. 1: Pseudocode of proposed tournament selection based random vector (TSRVDE) of DE algorithm

\section{Test functions and experimental results}

The performance of proposed TPSRVDE is accessed by taking a comprehensive set of $37 \mathrm{~N}$ dimensional benchmark functions. These benchmark functions are commonly used multidimensional global optimization problems that are given in the appendix section of this paper containing all necessary detail. Performance parameter Number of Function Calls (NFC) is one the most commonly used metric in evolutionary algorithms (Islam et al., 2012; Rahnamayan et al., 2008; Zhou et al., 2013) is used to measure the convergence speed of conventional DE mutations strategies and TSRVDE strategies. The success rate (SR) and acceleration rate (AR) of all mutation strategies are also calculated for analysis. 
The convergence speed of conventional and proposed mutation strategies is measure by using acceleration rate that is based on NFC. AR is calculated by using the Eq. 5

$$
A R=\frac{N F C_{D E}}{N F C_{T S R V D E}}
$$

TSRVDE is faster than DE if $A R>1, A R<1$ means that DE is faster than TSRVDE $A R=1$ will shows that both TSRVDE and DE possess the equivalent convergence speed. If an algorithm reaches to VTR (value to reach) then it is called successful and SR is measured based on number of times algorithm successfully reached to VTR. SR is calculated by using the following Eq. 6

$S R=\frac{\text { number of times reached } V T R}{\text { total number of trials }}$

Average acceleration rate for the test suit of benchmark functions is calculated by using Eq. 7 . Average acceleration rate (A. A. R)

$A R_{\text {ave }}=\frac{1}{n} \sum_{i=1}^{n} A R_{I}$

and average success rate for the test suit of benchmark functions is calculated by using Eq. 8 .

Average success rate (A.S.R) is given as follows (Eq. 8)

$$
A R_{\text {ave }}=\frac{1}{n} \sum_{i=1}^{n} A R_{I}
$$

NFC experimental results over the test suit of benchmark functions are given in this section. 30 independent trials are performed to generate the NFC results for binomial and exponential schemes of DE and TRSVDE mutation strategies. Experimental results show best values as bold faces that are contained in Tables 3, 4, and 5. Convergence graphs of DE and TSRVDE mutation strategies are shown in Fig. 2 and Fig. 3. TSRVDE mutation strategies are reported as TSRVDE 1 - TSRVDE 10 and standard DE mutation strategies are reported as $\mathrm{DE}_{1}-\mathrm{DE}_{10}$ in the results. Results are generated by using population size of 30, dimensions as 10D,20D, 30D and control parameters mutation probability and crossover rate as $\mathrm{F}=0.5, \mathrm{CR}=0.9$ (Storn and Price, 1997; MezuraMontes et al., 2006; Brest et al., 2006). Number of function calls value is calculated by taking 30 trials for both DE and TSRVDE for maximum NFC $10^{4 * D I M}$ (Wang et al., 2012). To find out NFC, VTR value is set to 0.0001 and Max-NFC values are 100,$000 ; 200,000$ and 300,000 for $10 \mathrm{D}, 20 \mathrm{D}$ and $30 \mathrm{D}$ respectively for both DE and TSRVDE in all functions.

Experimental results reported in Tables 3, 4, and 5 are obtained by using parameter setting given in section-IV of this paper. Experimental result contains NFC, AR and SR parameter results. Results of 10D, 20D and 30D are given in Table 3, Table 4 and Table 5 respectively. Result of each DE mutation strategy and its corresponding TSRVDE strategy are shown as a pair under binomial and exponential schemes. Table entries filled with dash (-) value shows the failure of mutation strategy to reach to VTR and values reported as boldface shows the best value. For effective analysis of research results, summary of results is presented in Table 6 .

Summary if experimental results are given in Table 6 that contains DE mutation strategies and its corresponding TSRVDE mutation strategies. The summary is given for $10 \mathrm{D}, 20 \mathrm{D}$ and $30 \mathrm{D}$ where column best shows number of times DE or TSRVDE mutation strategy has best NFC in Tables 3, 4, and 5 . Average success value for the test suit of benchmark functions is given as A.S.R and average acceleration rate as A.A.R in Table 6. It is clear from NFC results that TSRVDE1- TSRVDE 10 mutation strategies have dominating performance than $\mathrm{DE}_{1}-\mathrm{DE}_{10}$ in all cases. Similarly A.A.R shows that TSRVDE 1 - TSRVDE 10 mutation strategies are faster than their corresponding $\mathrm{DE}_{1}-\mathrm{DE}_{10}$ mutation strategies. A.S.R result shows that in few cases TSRVDE and DE mutation strategies have about similar performance, in few cases TSRVDE mutation strategies have slightly better performance than DE mutation strategies while in most of the cases DE has slightly better performance than TSRVDE mutation strategies.

Table 3: 10D-Number of functions calls (NFC), Success Rate (SR) and acceleration rate (AR) of DE and TSRVDE mutation

\begin{tabular}{|c|c|c|c|c|c|c|c|c|c|c|c|c|c|c|c|c|c|c|c|c|c|c|c|c|c|}
\hline \multirow{3}{*}{$\begin{array}{l}\text { : } \\
\text { 总 } \\
\text { 童 }\end{array}$} & \multicolumn{5}{|c|}{$\mathrm{DE} / \mathrm{rand} / 1 /$ bin } & \multicolumn{5}{|c|}{$\mathrm{DE} /$ best/1/bin } & \multicolumn{5}{|c|}{$\mathrm{DE} / \mathrm{rand}$ to best $/ 1 / \mathrm{bin}$} & \multicolumn{5}{|c|}{$\mathrm{DE} /$ rand/2/bin } & \multicolumn{5}{|c|}{$\mathrm{DE} /$ best/2/bin } \\
\hline & $\mathrm{DE}_{1}$ & & TSRVI & & & $\mathrm{DE}_{2}$ & & TSRVDE & & & $\mathrm{DE}_{3}$ & & $\begin{array}{l}\text { TSRVDE } \\
\end{array}$ & & & $\mathrm{DE}_{4}$ & & $\begin{array}{l}\text { TSRVDE } \\
\end{array}$ & & & $\mathrm{DE}_{5}$ & & TSRVD & & \\
\hline & NFC & S.R & NFC & S.R & A.R & NFC & S.R & NFC & S.R & A.R & $\mathrm{NFC}$ & S.R & NFC & S.R & A.R & NFC & S.R & NFC & S.R & A.R & $\mathrm{NFC}$ & S.R & NFC & S.R & A.R \\
\hline$f_{2}$ & 256.30 & 1 & 51.60 & 1 & 4.97 & 77.17 & 1 & 46.10 & 1 & 1.67 & 672.40 & 1 & 75.47 & 1 & 8.91 & 209.00 & 1 & 51.30 & 1 & 4.07 & 106.33 & 1 & 65.20 & 0.83 & 1.63 \\
\hline$f_{3}$ & 594.80 & 1 & 85.63 & 1 & 6.95 & 155.30 & 1 & 144.03 & 1 & 1.08 & 1910.43 & 1 & 138.97 & 1 & 13.75 & 444.97 & 1 & 85.47 & 1 & 5.21 & 213.10 & 1 & 70.73 & 1 & 3.01 \\
\hline$f_{4}$ & - & 0 & - & 0 & - & 1706.77 & 1 & - & 0 & - & 5755.33 & 1 & - & 0 & - & 1991.97 & 1 & 21026.13 & 1 & 0.09 & - & 0 & - & 0 & - \\
\hline$f_{5}$ & 13590.33 & 1 & 123.83 & 1 & 109.75 & 1350.13 & 1 & 78.48 & 0.97 & 17.20 & 79752.34 & 0.97 & 390.87 & 1 & 204.04 & 16324.20 & 1 & 189.87 & 1 & 85.98 & 5512.27 & 1 & 1616.97 & 1 & 3.41 \\
\hline$f_{6}$ & 9694.46 & 0.87 & 116.27 & 0.73 & 83.38 & 763.30 & 0.33 & 77.00 & 0.13 & 9.91 & & 0 & 264.15 & 0.67 & - & 69679.28 & 0.60 & 139.45 & 0.37 & 499.66 & 701.67 & 1 & 149.53 & 0.57 & 4.69 \\
\hline$f_{7}$ & 427.03 & 1 & 261.05 & 0.73 & 1.64 & 156.63 & 1 & 559.47 & 1 & 0.28 & 989.90 & 1 & 369.23 & 1 & 2.68 & 327.30 & 1 & 96.90 & 1 & 3.38 & 208.90 & 1 & 91.48 & 0.97 & 2.28 \\
\hline$f_{8}$ & 13389.60 & 1 & 150.11 & 0.90 & 89.20 & 1178.47 & 1 & 96.84 & 0.83 & 12.17 & 82356.21 & 0.97 & 417.30 & 1 & 197.35 & 16331.50 & 1 & 215.17 & 0.97 & 75.90 & 3977.20 & 1 & 2720.03 & 1 & 1.46 \\
\hline$f_{9}$ & 1062.00 & 1 & 157.96 & 0.93 & 6.72 & 275.80 & 1 & 61.83 & 0.97 & 4.46 & 2296.83 & 1 & 352.10 & 0.97 & 6.52 & 2011.07 & 1 & 177.17 & 0.97 & 11.35 & 483.40 & 1 & 314.33 & 1 & 1.54 \\
\hline$f_{10}$ & 510.60 & 1 & 81.23 & 1 & 6.29 & 112.60 & 1 & 49.77 & 1 & 2.26 & 1140.63 & 1 & 128.20 & 1 & 8.90 & 345.27 & 1 & 78.13 & 1 & 4.42 & 205.63 & 1 & 81.07 & 1 & 2.54 \\
\hline$f_{11}$ & 374.00 & 1 & 83.97 & 1 & 4.45 & 99.73 & 1 & 58.80 & 1 & 1.70 & 842.13 & 1 & 118.40 & 1 & 7.1 & 279.43 & 1 & 78.50 & 1 & 3.56 & 152.47 & 1 & 197.47 & 1 & 0.77 \\
\hline$f_{12}$ & 121.77 & 1 & 28.77 & 1 & 4.23 & 33.93 & 1 & 24.10 & 1 & 1.41 & 258.53 & 1 & 42.03 & 1 & 6.15 & 90.60 & 1 & 28.73 & 1 & 3.15 & 47.47 & 1 & 25.87 & 1 & 1.84 \\
\hline$f_{13}$ & - & 0 & - & 0 & - & 35629.10 & 0.97 & 33933.31 & 0.97 & 1.05 & - & 0 & - & 0 & - & - & 0 & - & 0 & - & 2678.00 & 0.27 & 942.43 & 0.47 & 2.84 \\
\hline$f_{14}$ & 96.40 & 1 & 21.67 & 1 & 4.45 & 25.53 & 1 & 15.33 & 1 & 1.67 & 212.73 & 1 & 28.77 & 1 & 7.40 & 69.43 & 1 & 20.13 & 1 & 3.45 & 37.83 & 1 & 16.10 & 1 & 2.35 \\
\hline$f_{15}$ & 12350.08 & 0.43 & 114.50 & 0.07 & 107.86 & 1855.50 & 0.07 & 84.00 & 0.23 & 22.09 & 40249.14 & 0.70 & 400.00 & 0.23 & 100.62 & 43475.27 & 0.73 & 322.33 & 0.30 & 134.88 & 34217.20 & 0.83 & 1604.33 & 0.20 & 21.33 \\
\hline$f_{16}$ & 54455.93 & 0.47 & 337.23 & 0.43 & 161.48 & 3983.67 & 0.10 & 618.82 & 0.37 & 6.44 & - & 0 & 2415.60 & 0.33 & - & - & 0 & 991.13 & 0.27 & - & - & 0 & 7216.50 & 0.07 & - \\
\hline$f_{17}$ & 7969.80 & 1 & 184.36 & 0.83 & 43.23 & 924.00 & 0.80 & 115.29 & 0.47 & 8.01 & - & 0 & 465.50 & 0.87 & - & 47533.07 & 1 & 268.00 & 0.77 & 177.36 & 15018.20 & 1 & 1320.46 & 0.87 & 11.37 \\
\hline$f_{18}$ & & 0 & - & 0 & & 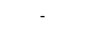 & 0 & & 0 & & - & 0 & & 0 & - & - & 0 & - & 0 & - & - & 0 & - & 0 & - \\
\hline$f_{19}$ & 336.00 & 1 & 58.90 & 1 & 5.70 & 80.30 & 1 & 44.03 & 1 & 1.82 & 819.13 & 1 & 94.13 & 1 & 8.70 & 254.17 & 1 & 57.27 & 1 & 4.44 & 142.57 & 1 & 82.04 & 0.87 & 1.74 \\
\hline$f_{22}$ & 199.37 & 1 & 42.70 & 1 & 4.67 & 52.70 & 1 & 33.83 & 1 & 1.56 & 471.33 & 1 & 61.10 & 1 & 7.71 & 158.27 & 1 & 39.00 & 1 & 4.06 & 77.97 & 1 & 469.00 & 1 & 0.17 \\
\hline
\end{tabular}
strategies 


\begin{tabular}{|c|c|c|c|c|c|c|c|c|c|c|c|c|c|c|c|c|c|c|c|c|c|c|c|c|c|}
\hline$f_{23}$ & 332.67 & 1 & 80.93 & 1 & 4.11 & 86.00 & 1 & 62.67 & 1 & 1.37 & 698.60 & 1 & $103 . C$ & 1 & 6.78 & 224.60 & 1 & 67.67 & 1 & 3.32 & 130.07 & 1 & 3977.27 & 1 & 0.03 \\
\hline$f_{24}$ & 176.93 & 1 & 39.57 & 1 & 4.47 & 45.87 & 1 & 33.00 & 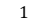 & 1.3 & 372.93 & 1 & 55.83 & 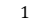 & 6.68 & 120.43 & 1 & 36.20 & 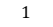 & 3.33 & 69.37 & 1 & 452.00 & 1 & 0.15 \\
\hline$f_{25}$ & 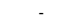 & 0 & & 0 & & 1435.00 & 0.1 & 12066.50 & 0.13 & 0.12 & & 0 & 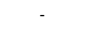 & 0 & & . & 0 & 1093.50 & 0.07 & - & & 0 & . & 0 & \\
\hline$f_{26}$ & 250.10 & 1 & 58.07 & 1 & 4.31 & 65.90 & 1 & 47.33 & 1 & 1.39 & 528.87 & 1 & 78.33 & 1 & 6.75 & 169.23 & 1 & 53.17 & 1 & 3.18 & 97.93 & 1 & 1639.90 & 1 & 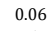 \\
\hline$f_{27}$ & 1535.87 & 1 & 360.43 & 1 & 4. & 30 & 1 & 202.30 & 1 & 1.8 & 3427.03 & 1 & 432.53 & 1 & 7.92 & 1006.83 & 1 & 267.73 & 1 & 3.76 & 770.47 & 1 & 298.00 & 0.07 & 2.59 \\
\hline$f_{28}$ & 286.20 & & 7.53 & 1 & 1. & 37 & 1 & 695.83 & 1 & 0. & 628.60 & 1 & 27 & 1 & .81 & 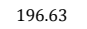 & 1 & 86.47 & 1 & 2.27 & 229.67 & 1 & 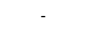 & 0 & \\
\hline$f_{29}$ & 976.87 & 1 & 101.60 & 0.17 & 9. & 6.44 & 0.53 & 82.83 & 0.20 & 11.31 & 1875 & 1 & 3.45 & 0.37 & 5.02 & 3 & 1 & 77.71 & 0.47 & 12.89 & .00 & 1 & 9.36 & 0.73 & \\
\hline$f_{30}$ & 295.40 & & & 1 & & & 1 & & 1 & 1.38 & & 1 & & 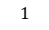 & & & 1 & 50 & 1 & 1.45 & & 1 & & 1 & \\
\hline$f_{31}$ & 287. & & & 1 & & & 1 & & 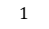 & & & 1 & & & & & 1 & & & & & 1 & & 1 & \\
\hline$f_{32}$ & 69.33 & 1 & & 1 & . & & 1 & & 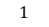 & 3. & 20 & 1 & & & & 12 & 1 & 18. & 1 & 6.5 & 27 & 1 & 3 & & \\
\hline$f_{33}$ & 6. & . & & 1 & & & 1 & & 1 & & & 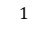 & & & & & 1 & & 2 & 0.80 & 9.1 & 1 & . & 1 & \\
\hline$f_{34}$ & 2006.5 & 1 & & 0 & 12 & 935.87 & 1 & 91.13 & 0.80 & 10.27 & 36802.00 & 0.77 & & 1 & 114.91 & & 1 & 232.81 & 0.90 & 79.37 & 279.40 & 1 & 275.40 & 1 & 1. \\
\hline$f_{35}$ & 3777. & in & & 0.97 & 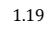 & - & 0 & 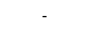 & 0 & . & - & 0 & & 1 & 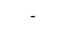 & & 0.40 & 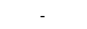 & 0 & - & - & 0 & - & 0 & \\
\hline$f_{36}$ & 30.33 & 1 & 27.17 & 1 & 1.12 & 73 & 1 & 28.23 & 1 & 1.48 & 18.43 & 1 & 22.83 & 1 & 0.81 & 22.43 & 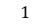 & 25.20 & 1 & 0.89 & 30. & 1 & 22.77 & 1 & 1. \\
\hline$f_{3}$ & 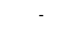 & 0 & 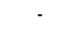 & 0 & & 2713.67 & 0.10 & & 0 & 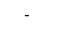 & & 0 & - & 0 & & 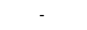 & 0 & & 0 & 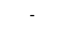 & 55579.33 & 0.60 & 5757.00 & 0.03 & \\
\hline
\end{tabular}

\begin{tabular}{|c|c|c|c|c|c|c|c|c|c|c|c|c|c|c|c|c|c|c|c|c|c|c|c|c|c|}
\hline \multirow{3}{*}{ int } & \multicolumn{5}{|c|}{ DE/rand/1/exp } & \multicolumn{5}{|c|}{ DE/best/1/exp } & \multicolumn{5}{|c|}{$\mathrm{DE} / \mathrm{rand}$ to best/1/exp } & \multicolumn{5}{|c|}{ DE/rand/2/exp } & \multicolumn{5}{|c|}{$\mathrm{DE} /$ best/2/exp } \\
\hline & $\mathrm{DE}_{6}$ & & TSRVDI & & & $\mathrm{DE}_{7}$ & & TSRVDI & & & $\mathrm{DE}_{8}$ & & TSRVDE & & & $\mathrm{DE}_{9}$ & & TSRVDE & & & $\mathrm{DE}_{10}$ & & TSRVDE & & \\
\hline & $\mathrm{NFC}$ & S.R & $\mathrm{NFC}$ & S.R & A.R & $\mathrm{NFC}$ & S.R & $\mathrm{NFC}$ & S.R & A.R & $\mathrm{NFC}$ & S.R & $\mathrm{NFC}$ & S.R & A.R & $\mathrm{NFC}$ & S.R & $\mathrm{NFC}$ & S.R & A.R & $\mathrm{NFC}$ & S.R & $\mathrm{NFC}$ & S.R & A.R \\
\hline$f_{1}$ & 158.20 & 1 & 117.13 & 1 & 1.35 & 124.33 & 1 & 103.23 & 1 & 1.20 & 191.47 & 1 & 135.17 & 1 & 1.42 & 160.60 & 1 & 118.90 & 1 & 1.35 & 178.90 & 1 & 154.70 & 1 & 1.16 \\
\hline$f_{2}$ & 170.47 & 1 & 122.77 & 1 & 1.39 & 129.90 & 1 & 107.90 & 1 & 1.20 & 205.77 & 1 & 140.90 & 1 & 1.46 & 169.20 & 1 & 123.10 & 1 & 1.37 & 196.17 & 1 & 163.00 & 1 & 1.20 \\
\hline$f_{3}$ & 6768.33 & 1 & 3115.96 & 0.93 & 2.17 & 4922.43 & 1 & 3254.57 & 0.70 & 1.51 & 7970.50 & 1 & 3711.33 & 1 & 2.15 & 6665.30 & 1 & 2993.53 & 1 & 2.23 & 3880.67 & 1 & 2136.14 & 0.70 & 1.82 \\
\hline$f_{5}$ & 261.47 & 1 & 183.96 & 0.87 & 1.42 & 203.83 & 1 & 165.33 & 0.70 & 1.23 & 340.77 & 1 & 220.27 & 1 & 1.55 & 279.10 & 1 & 193.66 & 0.97 & 1.44 & 326.07 & 1 & 276.53 & 1 & 1.18 \\
\hline$f_{6}$ & 592.03 & 1 & 407.04 & 0.90 & 1.45 & 2607.95 & 0.70 & 749.75 & 0.27 & 3.48 & 848.47 & 1 & 521.63 & 1 & 1.63 & 715.90 & 0.97 & 1026.08 & 0.87 & 0.70 & 643.40 & 1 & 652.24 & 0.97 & 0.99 \\
\hline$f_{7}$ & 138.00 & 1 & 67.17 & 1 & 2.05 & 91.70 & 1 & 57.21 & 0.97 & 1.60 & 164.50 & 1 & 76.90 & 1 & 2.14 & 128.57 & 1 & 65.27 & 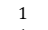 & 1.97 & 157.33 & 1 & 102.00 & 1 & 1.54 \\
\hline$f_{8}$ & 338.90 & 1 & 228.92 & 0.83 & 1.48 & 255.53 & 1 & 234.73 & 0.50 & 1.09 & 432.70 & 1 & 279.20 & 1 & 1.55 & 351.03 & 1 & 244.17 & 1 & 1.44 & 414.17 & 1 & 357.72 & 0.97 & 1.16 \\
\hline$f_{9}$ & 107.13 & 1 & 93.13 & 1 & 1.15 & 94.27 & 1 & 78.30 & 1 & 1.20 & 125.00 & 1 & 94.10 & 1 & 1.33 & 107.53 & 1 & 90.73 & 1 & 1.19 & 120.23 & 1 & 106.77 & 1 & 1.13 \\
\hline$f_{10}$ & 22296.43 & 1 & 11016.79 & 0.97 & 2.02 & 19522.83 & 1 & 16493.78 & 0.77 & 1.18 & 22663.67 & 1 & 11432.87 & 1 & 1.98 & 22905.53 & 1 & 12053.83 & 1 & 1.90 & 11755.63 & 1 & 6637.77 & 1 & 1.77 \\
\hline$f_{11}$ & 301.50 & 1 & 215.90 & 0.97 & 1.40 & 235.53 & 1 & 193.15 & 0.90 & 1.22 & 373.30 & 1 & 253.00 & 1 & 1.48 & 308.67 & 1 & 226.37 & 1 & 1.36 & 354.03 & 1 & 301.73 & 1 & 1.17 \\
\hline$f_{12}$ & 108.40 & 1 & 79.47 & 1 & 1.36 & 84.50 & 1 & 71.17 & 1 & 1.19 & 128.07 & 1 & 91.83 & 1 & 1.39 & 108.07 & 1 & 83.23 & 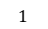 & 1.30 & 117.03 & 1 & 104.20 & 1 & 1.12 \\
\hline$f_{13}$ & - & 0 & - & 0 & - & - & 0 & - & 0 & - & - & 0 & - & 0 & - & - & 0 & & 0 & - & & 0 & - & 0 & - \\
\hline$f_{14}$ & 67.43 & 1 & 52.53 & 1 & 1.28 & 54.73 & 1 & 46.43 & 1 & 1.18 & 80.47 & 1 & 59.30 & 1 & 1.36 & 67.10 & 1 & 53.60 & 1 & 1.25 & 75.87 & 1 & 63.60 & 1 & 1.19 \\
\hline$f_{15}$ & 5459.40 & 1 & 4800.03 & 1.00 & 1.14 & 8479.97 & 1 & 11287.74 & 0.90 & 0.75 & 5296.03 & 1 & 2952.47 & 1 & 1.79 & 4160.77 & 1 & 7002.37 & 1 & 0.59 & 3295.00 & , & 3629.30 & 1 & 0.91 \\
\hline$f_{16}$ & - & 0 & & 0 & - & (2) & 0 & - & 0 & - & - & 0 & 55305.00 & 0.03 & . & - & 0 & 73029.00 & 0.03 & - & & 0 & - & 0 & . \\
\hline$f_{17}$ & 2028.83 & 1 & 488.87 & 0.77 & 4.15 & 992.96 & 0.87 & 473.57 & 0.23 & 2.10 & 4966.67 & 1 & 700.03 & 1 & 7.09 & 2554.27 & 1 & 590.86 & 0.93 & 4.32 & 3332.50 & 1 & 983.40 & 1 & 3.39 \\
\hline$f_{18}$ & 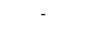 & 0 & & 0 & - & & 0 & 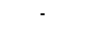 & 0 & - & - & 0 & & 0 & & & 0 & & 0 & - & & 0 & 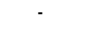 & 0 & - \\
\hline$f_{19}$ & 150.33 & 1 & 105.03 & 1 & 1.43 & 113.00 & 1 & 94.62 & 0.97 & 1.19 & 186.90 & 1 & 122.70 & 1 & 1.52 & 152.57 & 1 & 109.73 & 1 & 1.39 & 179.67 & 1 & 147.73 & 1 & 1.22 \\
\hline$f_{20}$ & 42374.07 & 1 & 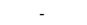 & 0 & - & 40842.70 & 1 & - & 0 & - & 21337.30 & 1 & 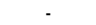 & 0 & - & 50160.00 & 1 & 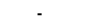 & 0 & - & & 0 & - & 0 & - \\
\hline$f_{21}$ & - & 0 & - & 0 & - & 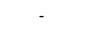 & 0 & - & 0 & - & 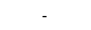 & 0 & - & 0 & - & - & 0 & 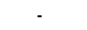 & 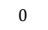 & - & & 0 & - & 0 & - \\
\hline$f_{22}$ & 156.40 & 1 & 112.93 & 1 & 1.38 & 121.03 & 1 & 102.00 & 1 & 1.19 & 193.00 & 1 & 131.13 & 1 & 1.47 & 159.33 & 1 & 118.40 & 1 & 1.35 & 199.37 & 1 & 170.60 & 1 & 1.17 \\
\hline$f_{25}$ & 1899.17 & 0.97 & 609.63 & 0.27 & 3.12 & 1748.72 & 0.60 & 1662.00 & 0.23 & 1.05 & 6795.50 & 1 & 986.48 & 0.90 & 6.89 & 5228.08 & 0.87 & 697.54 & 0.43 & 7.50 & 2462.29 & 0.93 & 8493.73 & 0.50 & 0.29 \\
\hline$f_{26}$ & 218.93 & 1 & 161.07 & 1 & 1.36 & 170.10 & 1 & 140.43 & 1 & 1.21 & 264.97 & 1 & 181.80 & 1 & 1. & 219.20 & 1 & 164.57 & 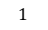 & 1. & 253.03 & 1 & 216.37 & 1 & 1.17 \\
\hline$f_{27}$ & 232.57 & 1 & 165.57 & 1 & 1. & 187.80 & 1 & 147.90 & 0.97 & 1.27 & 285.13 & 1 & 191.70 & 1 & 1. & 249.03 & 1 & 169.70 & $1+$ & & 273.97 & 1 & 235.80 & 1 & 1.16 \\
\hline$f_{2}$ & 700.03 & 1 & 274.67 & 0.50 & 2.55 & 345.80 & 1 & 612.15 & 0.43 & 0.56 & 724.80 & 1 & 379.20 & 1 & 1. & 560.4 & 1 & 288.30 & 1 & 1.94 & 655.24 & 0.70 & 340.05 & 0.63 & 1.93 \\
\hline$f_{29}$ & 153.37 & 1 & 141.10 & 1 & 1.09 & 159.07 & 1 & 134.80 & 1 & 1.18 & 210.70 & 1 & 165.73 & 1 & 1.27 & 210.63 & 1 & 161.33 & 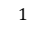 & 1.31 & 147.00 & 1 & 132.50 & 1 & 1.11 \\
\hline$f_{30}$ & 235.70 & 1 & 183.33 & 1 & 1.29 & 180.13 & 1 & 165.07 & 1 & 1.09 & 278.47 & 1 & 192.87 & 1 & 1. & 223.70 & 1 & 171.83 & 1 & 1.30 & 414.07 & 1 & 376.87 & 1 & 1.10 \\
\hline$f_{31}$ & 238.10 & 1 & 187.13 & 1 & 1.27 & 187.47 & 1 & 182.40 & 1 & 1.03 & 281.33 & 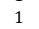 & 200.40 & 1 & 1. & 227.60 & 1 & 179.93 & 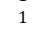 & 1.26 & 416.27 & 1 & 388.07 & 1 & 1.07 \\
\hline$f_{32}$ & 36.17 & 1 & 28.23 & 1 & 1.2 & 33.67 & 1 & 25.80 & 1 & 1. & 43.27 & 1 & 34. & 1 & 1. & 39.57 & 1 & 31.90 & 1 & & 36.47 & 1 & 31.77 & 1 & 1.15 \\
\hline$f_{33}$ & 8.23 & 1 & 6.13 & 1 & 1.3 & 7.50 & 1 & 6.80 & 1 & 1.10 & 8.67 & 1 & 6. & 1 & 1. & 6.1 & 1 & 6.57 & 1 & 0.93 & 11.37 & 1 & 8.47 & 1 & 1.34 \\
\hline$f_{34}$ & 495.93 & 1 & 286.50 & 0.67 & 1.7 & 305.93 & 1 & 236.74 & 0.63 & 1.29 & 751.57 & 1 & 381.50 & 1 & 1. & 489.20 & 1 & 297.63 & 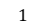 & 1.64 & 698.97 & 1 & 522.90 & 1 & 1.34 \\
\hline$f_{35}$ & 1668.00 & 1 & 587.00 & 1 & 2.8 & 675.29 & 0.23 & 493.50 & 0.07 & 1.37 & 176 & - & & 1 & 20. & 1286.13 & 1 & 767.89 & 0.93 & 1.67 & 1334.04 & 0.90 & 775.62 & 0.97 & 1.72 \\
\hline$f_{36}$ & 126.07 & 1 & 170.47 & 1 & 0.74 & 110.67 & 1 & 70.92 & 0.83 & 1.56 & 109.63 & 1 & 110.27 & 1 & 0.99 & 128.70 & 1 & 82.28 & 0.97 & 1.56 & 80.90 & 1 & 119.77 & 1 & 0.68 \\
\hline$f_{37}$ & & 0 & & 0 & . & & 0 & 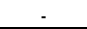 & 0 & & & 0 & 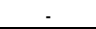 & 0 & & & 0 & & 0 & - & & 0 & . & 0 & \\
\hline
\end{tabular}

Table 4: 20D-Number of functions calls (NFC), Success Rate (SR) and acceleration rate (AR) of DE and TSRVDE mutation strategies

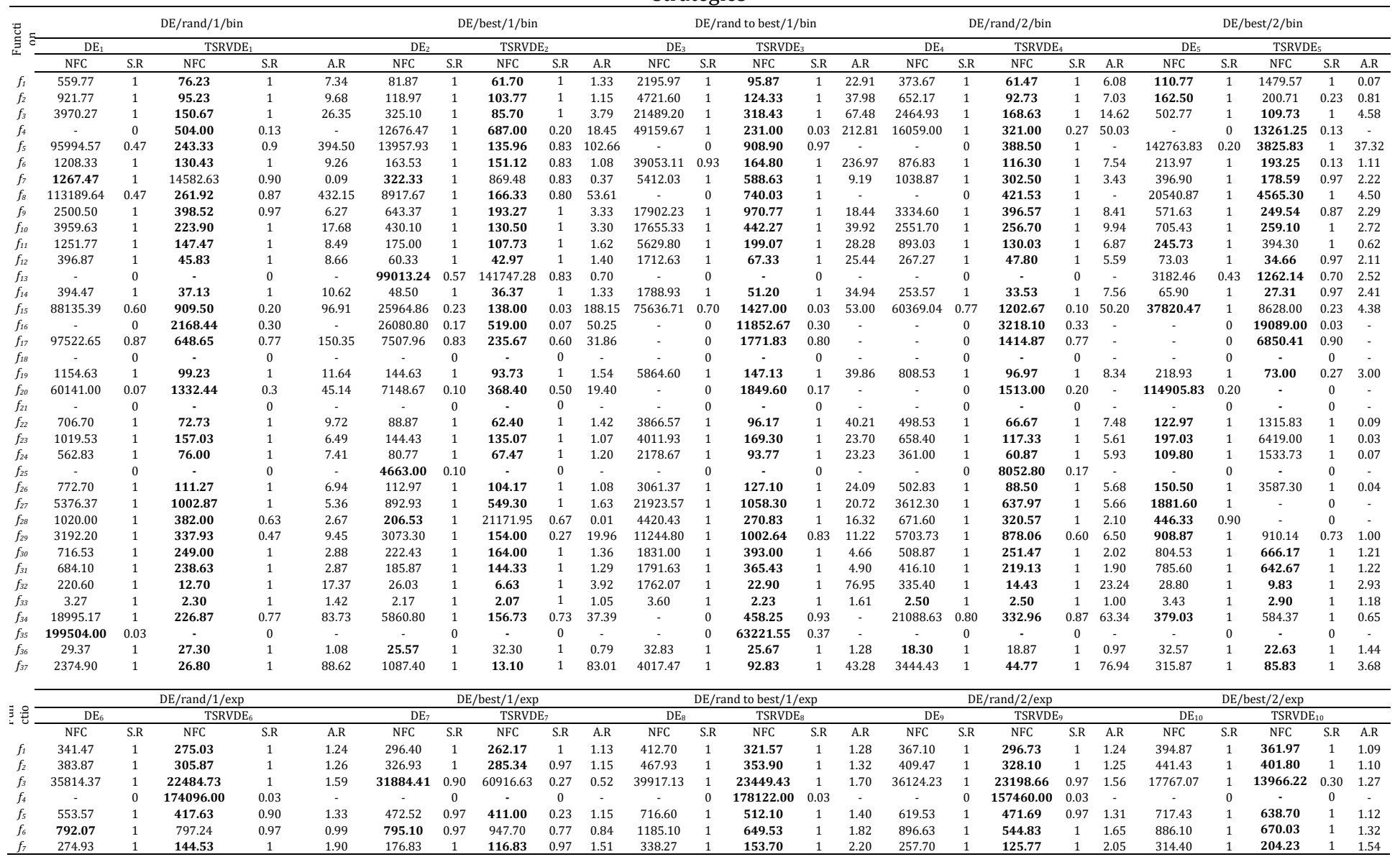




\begin{tabular}{|c|c|c|c|c|c|c|c|c|c|c|c|c|c|c|c|c|c|c|c|c|c|c|c|c|c|}
\hline $\begin{array}{l}f_{8} \\
\end{array}$ & 691.60 & 1 & 530.44 & 0.83 & 1.30 & 576.33 & 1 & 515.40 & 0.33 & 1.12 & 881.80 & 1 & 632.50 & 1 & 1.39 & 755.30 & 1 & 578.97 & 1 & 1.30 & 877.93 & 1 & 777.67 & 1 & 1.13 \\
\hline$f_{9}$ & 196.87 & 1 & 165.83 & 1 & 1.19 & 172.40 & 1 & 151.50 & 1 & 1.14 & 228.07 & 1 & 190.87 & 1 & 1.19 & 205.60 & 1 & 177.30 & 1 & 1.16 & 214.00 & 1 & 195.43 & 1 & 1.10 \\
\hline$f_{10}$ & & 0 & - & 0 & - & - & 0 & - & 0 & 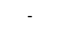 & - & 0 & & 0 & - & - & 0 & - & 0 & 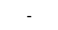 & 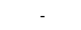 & 0 & - & 0 & 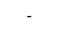 \\
\hline$f_{11}$ & 640.53 & 1 & 503.17 & 1 & 1.27 & 548.20 & 1 & 467.78 & 0.90 & 1.17 & 784.67 & 1 & 590.03 & 1 & 1.33 & 698.20 & 1 & 550.60 & 1 & 1.27 & 760.30 & 1 & 680.70 & 1 & 1.12 \\
\hline$f_{12}$ & 235.23 & 1 & 194.87 & 1 & 1.21 & 201.43 & 1 & 181.40 & 1 & 1.11 & 280.27 & 1 & 222.27 & 1 & 1.26 & 246.80 & 1 & 207.30 & 1 & 1.19 & 260.80 & 1 & 245.80 & 1 & 1.06 \\
\hline$f_{13}$ & & 0 & 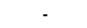 & 0 & & & 0 & 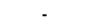 & 0 & & & 0 & & 0 & & & 0 & & 0 & & & 0 & 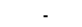 & 0 & \\
\hline$f_{14}$ & 170.30 & 1 & 143.43 & 1 & 1.19 & 148.23 & 1 & 134.07 & 1 & 1.11 & 201.87 & 1 & 158.47 & 1 & 1.27 & 179.30 & 1 & 147.40 & 1 & 1.22 & 183.70 & 1 & 177.63 & 1 & 1.03 \\
\hline$f_{15}$ & 5630.40 & 1 & 3883.33 & 1 & 1.45 & 4027.10 & 1 & 6396.50 & 1 & 0.63 & 5567.03 & 1 & 4819.97 & 1 & 1.15 & 4962.97 & 1 & 4573.93 & 1 & 1.09 & 3378.93 & 1 & 4342.33 & 1 & 0.78 \\
\hline$f_{16}$ & & 0 & - & 0 & ( & & 0 & 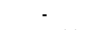 & 0 & & & 0 & & 0 & & - & 0 & & 0 & & 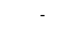 & 0 & - & 0 & 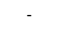 \\
\hline$f_{17}$ & 4615.97 & 1 & 1757.57 & 0.47 & 2.63 & 3842.74 & 0.90 & 1998.55 & 0.37 & 1.92 & 11055.97 & 1 & 2322.87 & 1 & 4.76 & 6852.43 & 1 & 953.00 & 0.77 & 3.51 & 7471.93 & 1 & 2857.23 & 1 & 2.62 \\
\hline$f_{18}$ & & 0 & - & 0 & & & 0 & . & 0 & - & . & 0 & & 0 & - & . & 0 & . & 0 & & . & 0 & - & 0 & 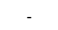 \\
\hline$f_{19}$ & 306.73 & 1 & 367 & 1 & 1.30 & 54.63 & 1 & 25,07 & 0.97 & 1.13 & 376.60 & 1 & 79.0 & 1 & 1.35 & 328.70 & 1 & 259.0 & 1 & 1.27 & 365.30 & 1 & 316.30 & 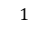 & 1.15 \\
\hline$f_{20}$ & 61102.36 & 0.93 & 2402.4 & 1 & 25.43 & 60791.09 & 0.77 & 2508.83 & 1 & 24.23 & 92402.04 & 0.87 & 2154.50 & 1 & 42.89 & 67923.29 & 0.93 & 2822.80 & 1 & 24.06 & 35618.86 & 0.97 & 1010.77 & 1 & 35.24 \\
\hline$f_{21}$ & - & 0 & . & 0 & - & - & 0 & - & 0 & - & - & 0 & - & 0 & - & - & 0 & - & 0 & - & - & 0 & (hes & 0 & - \\
\hline$f_{22}$ & 339.27 & 1 & 268.5 & 1 & 1.26 & 290.43 & 1 & 269.2 & 1 & 1.08 & 417.23 & 1 & 314.03 & 1 & 1.33 & 365.43 & 1 & 289.37 & 1 & 1.26 & 456.43 & 1 & 401.97 & 1 & 1.14 \\
\hline$f_{23}$ & 604.50 & 1 & 487.30 & 1 & 1.24 & 7.70 & 1 & 457.40 & 1 & 1.13 & 740.37 & 1 & 567.13 & 1 & 1.31 & 653.20 & 1 & 520.83 & 1 & 1.2 & 713.10 & 1 & 642.33 & 1 & 1.11 \\
\hline$f_{24}$ & 336.83 & 1 & $270-1$ & 1 & 122 & & 1 & 251.77 & 1 & 1.16 & 407.87 & 1 & 313.37 & 1 & 1.30 & 359.53 & 1 & & 1 & & 389.10 & 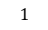 & 355.77 & 1 & 1.09 \\
\hline$f_{25}$ & 22617.00 & 0.73 & 64188.50 & 0.07 & 0.35 & 3859.50 & 0.27 & (1) & 0 & - & 30562.08 & 0.43 & 14352.75 & 0.40 & 2.13 & 14917.75 & 0.13 & 6469.29 & 0.23 & 2.31 & 8499.96 & 0.77 & 9752.13 & 0.27 & 0.87 \\
\hline$f_{26}$ & 464.63 & 1 & 373.33 & 1 & 1.24 & 397.80 & 1 & 349.47 & 1 & 1.14 & 568.90 & 1 & 432.57 & 1 & 1.3 & 496.67 & 1 & 404.13 & 1 & 1.2 & 541.67 & 1 & 488.57 & 1 & 1.11 \\
\hline$f_{27}$ & 483.77 & 1 & 382.67 & 1 & 1.26 & 419.13 & 1 & 359.55 & 0.97 & 1. & 591 & 1 & 442 & 1 & 1. & 533.03 & 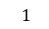 & 408 & 1 & 1. & 566.93 & 1 & 513.60 & 1 & 1.10 \\
\hline$f_{28}$ & 2604.23 & 1 & - & 0 & 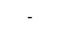 & 1449.67 & 1 & 16623.50 & 0.07 & 0.09 & 2278.10 & 1 & 1541.27 & 1 & 1.4 & 2406.73 & 1 & 1233.80 & 1 & 1.95 & 2162.00 & 0.03 & 1160.00 & 0.10 & 1.86 \\
\hline$f_{29}$ & 172.70 & 1 & 158. & 1 & 1.09 & 153.47 & 1 & 150.43 & 1 & 1.02 & 208.70 & 1 & 173.53 & 1 & 1.2 & 186.13 & 1 & 169.73 & 1 & 1.10 & 150.40 & 1 & 127.00 & 1 & 1.18 \\
\hline$f_{30}$ & 509.50 & 1 & 4 & 1 & 1. & 44 & 1 & 4 & 1 & 1.0 & 600.33 & 1 & 47 & 1 & 1. & 25.57 & 1 & 441 & 1 & 1. & 896.77 & 1 & 863.41 & 0.97 & 1.04 \\
\hline$f_{31}$ & 515.57 & 1 & 43 & 0.97 & & 44 & 1 & & 1 & 1. & 600 & 1 & 47 & 1 & 1 & 52 & 1 & 45 & 1 & 1 & $89 \mathrm{c}$ & 1 & 863.83 & 0.97 & 1.04 \\
\hline$f_{32}$ & 57.93 & 1 & 48.07 & 1 & 1. & 52.70 & 1 & 46 & 1 & 1. & 67.3 & 1 & 54.30 & 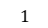 & 1.2 & 64.10 & 1 & 53.9 & 1 & 1. & 54.77 & 1 & 52.63 & 1 & 1.04 \\
\hline$f_{33}$ & 3.2 & 1 & & 1 & & & 1 & & 1 & & 4. & 1 & & 1 & & 3. & 1 & 3. & 1 & & 4.07 & 1 & 3.57 & 1 & 1.14 \\
\hline$f_{34}$ & 1037.07 & 1 & 659.2 & 0.60 & 1. & 714.87 & 1 & 595.07 & 0.47 & 1.20 & 1598.30 & 1 & 892.07 & 1 & 1.79 & 1095.00 & 1 & 728.40 & 1 & 1. & 1587.63 & 1 & 1220.77 & 1 & 1.30 \\
\hline$f_{35}$ & 11471 & 1 & & -2 & & - & 0 & - & 0 & - & - & 0 & 491 & 1 & ( & 59 & 0.67 & 303 & 0.40 & 1 & 0 & 0.03 & 2832.00 & 0.17 & 2.79 \\
\hline$f_{36}$ & 198.30 & 1 & 18 & 0.97 & 1 & 178 & 1 & 93 & 0.83 & 1.9 & 175.63 & 1 & 20 & 1 & 0.8 & 231.27 & 1 & 171.10 & 1 & 1. & 144.17 & 1 & 201.97 & 1 & 0.71 \\
\hline$f_{37}$ & 31.40 & 1 & 29.90 & 1 & 1.05 & 33.27 & 1 & 30.23 & 1 & 1.10 & 32.00 & 1 & 31.73 & 1 & 1.01 & 32.90 & 1 & 33.80 & 1 & 0.97 & 31.17 & 1 & 33.23 & 1 & 0.94 \\
\hline
\end{tabular}

Table 5: 30D-Number of functions calls (NFC), Success Rate (SR) and acceleration rate (AR) of DE and TSRVDE mutation

strategies

\begin{tabular}{|c|c|c|c|c|c|c|c|c|c|c|c|c|c|c|c|c|c|c|c|c|c|c|c|}
\hline & & & $\mathrm{DE} / \mathrm{rand} / 1$ & & & & & /best/1/bin & & & & $\mathrm{DE} / \mathrm{ran}$ & id to best $/ 1 / \mathrm{b}$ & & & & $\mathrm{DE} / \mathrm{L}$ & rand/2/bin & & & & $\mathrm{DE} / \mathrm{l}$ & best/2/bin \\
\hline$\Xi$ & $\mathrm{DE}_{1}$ & & TSR & & & $\mathrm{DE}_{2}$ & & TSRVDE & & & $\mathrm{DE}_{3}$ & & TSRVDE & & & $\mathrm{DE}_{4}$ & & TSRVDH & & & $\mathrm{DE}_{5}$ & & TSRVDE \\
\hline & NFC & $\begin{array}{ll}S . R \\
\end{array}$ & NFC & $\begin{array}{ll}S . R \\
\end{array}$ & A.R & NFC & $\begin{array}{ll}S . R \\
\end{array}$ & NFC & $\begin{array}{ll}S . R \\
\end{array}$ & A.R & NFC & S.R & NFC & S.R & A.R & NFC & S.R & NFC & S.R & A.R & NFC & S.R & NFC \\
\hline$f_{1}$ & 1298.50 & 1 & 114.80 & 1 & 11.31 & 111.73 & 1 & 99.53 & 1 & 1.12 & 9655.23 & 1 & 129.63 & 1 & 74.48 & 682.37 & 1 & $\begin{array}{l}88.67 \\
88\end{array}$ & 1 & 7.70 & 139.47 & 1 & 2574.97 \\
\hline$f_{2}$ & 2318.17 & 1 & 158.53 & 1 & 14.62 & 162.50 & 1 & 216.83 & 1 & 0.75 & 24435.57 & 1 & 178.37 & 1 & 137.00 & 1179.53 & 1 & 135.10 & 1 & 8.73 & 212.93 & 1 & 509.50 \\
\hline$f_{3}$ & 17057.93 & 1 & 210.43 & 1 & 81.06 & 579.00 & 1 & 116.17 & 1 & 4.98 & 213556.47 & 1 & 507.93 & 1 & 420.44 & 7922.73 & 1 & 258.33 & 1 & 30.67 & 988.53 & 1 & 138.40 \\
\hline$f_{4}$ & - & 0 & 869.50 & 0.27 & - & 43165.27 & 1 & 892.33 & 0.20 & 48.37 & 224129.00 & 1 & 576.00 & 0.13 & 389.11 & 71018.00 & 1 & 528.60 & 0.33 & 134.35 & - & 0 & 19867.67 \\
\hline$f_{5}$ & 296494.50 & 0.07 & 356.77 & 0.87 & 831.05 & 51471.16 & 0.83 & 198.79 & 0.93 & 258.93 & & 0 & 1150.87 & 1 & & & 0 & 540.13 & 1 & & 202158.00 & 0.03 & 5566.70 \\
\hline$f_{6}$ & 2976.47 & 1 & 239.70 & 1 & 12.42 & 223.83 & 0.97 & 312.57 & 0.93 & 0.72 & 31084.03 & 1 & 234.10 & 1 & 132.78 & 1606.40 & 1 & 194.07 & 1 & 8.28 & 302.00 & 1 & 76.00 \\
\hline$f_{7}$ & 2346.60 & 1 & 10673.33 & 0.80 & 0.22 & 485.67 & 1 & 4042.91 & 0.77 & 0.12 & 14704.50 & 1 & 3658.27 & 1 & 4.02 & 1877.20 & 1 & 1328.57 & 1 & 1.41 & 643.47 & 1 & 262.03 \\
\hline$f_{8}$ & 197627.80 & 0.17 & 350.04 & 0.83 & 564.59 & 55006.92 & 0.83 & 209.65 & 0.67 & 262.38 & & 0 & 1056.28 & 0.97 & & & 0 & 524.14 & 0.93 & - & 34102.80 & 1 & 6718.38 \\
\hline$f_{9}$ & 5186.50 & 1 & 513.43 & 1 & 10.10 & 1087.00 & 1 & 169.30 & 1 & 6.42 & 34859.63 & 1 & 1170.30 & 1 & 29.79 & 4564.90 & 1 & 654.77 & 1 & 6.97 & 791.90 & 1 & 793.75 \\
\hline$f_{10}$ & 15776.77 & 1 & 441.90 & 1 & 35.70 & 897.33 & 1 & 207.47 & 1 & 4.33 & 136575.77 & 1 & 941.63 & 1 & 145.04 & 8538.77 & 1 & 469.03 & 1 & 18.21 & 1510.37 & 1 & 328.43 \\
\hline$f_{11}$ & 3138.50 & 1 & 209.73 & 1 & 14.96 & 226.27 & 1 & 49.67 & 1 & 4.56 & 28561.30 & 1 & 267.57 & 1 & 106.74 & 1697.47 & 1 & 165.17 & 1 & 10.28 & 316.57 & 1 & 566.17 \\
\hline$f_{12}$ & 970.17 & 1 & 63.63 & 1 & 15.25 & 80.63 & 1 & 75.73 & 1 & 1.06 & 8493.67 & 1 & 87.97 & 1 & 96.56 & 503.27 & 1 & 63.53 & 1 & 7.92 & 94.27 & 1 & 73.88 \\
\hline$f_{13}$ & - & 0 & - & 0 & & 241401.67 & 0.10 & 174347.62 & 0.87 & 1.38 & & 0 & - & 0 & 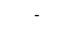 & & 0 & - & 0 & - & 4665.36 & 0.37 & 1167.14 \\
\hline$f_{14}$ & 1016.23 & 1 & 53.43 & 1 & 19.02 & 67.93 & 1 & 55.30 & 1 & 1.23 & 9867.63 & 1 & 66.90 & 1 & 147.50 & 485.20 & 1 & 47.47 & 1 & 10.22 & 89.20 & 1 & 119.54 \\
\hline$f_{15}$ & 115262.30 & 0.77 & 2682.00 & 0.10 & 42.98 & 86439.32 & 0.63 & 4203.00 & 0.03 & 20.57 & 147618.85 & 0.67 & 9813.38 & 0.27 & 15.04 & 106050.19 & 0.70 & 2629.57 & 0.23 & 40.33 & 84507.17 & 0.97 & 26709.68 \\
\hline$f_{16}$ & - & 0 & 4979.00 & 0.20 & - & 264140.00 & 0.03 & 2935.00 & 0.03 & 90.00 & - & 0 & 19029.25 & 0.4 & - & - & 0 & 3857.00 & 0.13 & - & 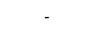 & 0 & 10582.00 \\
\hline$f_{17}$ & 230887.67 & 0.2 & 989.54 & 0.80 & 233.33 & 38826.41 & 0.73 & 1045.47 & 0.57 & 37.14 & - & 0 & 4262.11 & 0.93 & - & - & 0 & 2251.47 & 0.63 & - & - & 0 & 18525.26 \\
\hline$f_{18}$ & 33175.60 & 1 & 54.57 & 1 & 607.98 & 1391.43 & 1 & 25.87 & 1 & 53.79 & - & 0 & 237.13 & 1 & - & - & 0 & 97.13 & 1 & - & 902.00 & 0.17 & 842.17 \\
\hline$f_{19}$ & 2724.27 & 1 & 166.33 & 1 & 16.38 & 184.07 & 1 & 155.87 & 1 & 1.18 & 29459.50 & 1 & 197.10 & 1 & 149.46 & 1464.30 & 1 & 133.47 & 1 & 10.97 & 283.03 & 1 & 327.00 \\
\hline$f_{20}$ & - & 0 & 5743.75 & 0.13 & - & 44450.80 & 0.17 & 2352.67 & 0.10 & 18.89 & - & 0 & 14454.60 & 0.17 & - & 82317.00 & 0.07 & 6237.33 & 0.20 & 13.20 & 117162.00 & 0.17 & 3710.20 \\
\hline$f_{21}$ & - & 0 & - & 0 & - & & 0 & - & 0 & & & 0 & & 0 & & & 0 & - & 0 & - & & 0 & 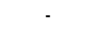 \\
\hline$f_{22}$ & 1756.40 & 1 & 117.17 & 1 & 14.99 & 117.63 & 1 & 96.30 & 1 & 1.22 & 25316.03 & 1 & 127.73 & 1 & 198.19 & 893.23 & 1 & 87.07 & 1 & 10.26 & 153.30 & 1 & 2427.87 \\
\hline$f_{23}$ & 2328.63 & 1 & 241.43 & 1 & 9.65 & 202.63 & 1 & 214.80 & 1 & 0.94 & 17286.20 & 1 & 234.53 & 1 & 73.70 & 1214.17 & 1 & 175.27 & 1 & 6.93 & 247.90 & 1 & 8574.23 \\
\hline$f_{24}$ & 1302.43 & 1 & 111.20 & 1 & 11.71 & 108.47 & 1 & 93.87 & 1 & 1.16 & 9579.90 & 1 & 124.20 & 1 & 77.13 & 667.47 & 1 & 86.07 & 1 & 7.76 & 139.53 & 1 & 2647.73 \\
\hline$f_{25}$ & - & 0 & - & 0 & - & 11080.00 & 0.13 & - & 0 & - & - & 0 & 166059.00 & 0.03 & - & - & 0 & 29882.25 & 0.13 & - & (2) & 0 & - \\
\hline$f_{26}$ & 1778.73 & 1 & 182.50 & 1 & 9.75 & 151.50 & 1 & 152.63 & 1 & 0.99 & 13265.03 & 1 & 180.53 & 1 & 73.48 & 922.43 & 1 & 126.53 & 1 & 7.29 & 191.27 & 1 & 5395.47 \\
\hline$f_{27}$ & 10921.60 & 1 & 1945.27 & 1 & 5.61 & 1416.27 & 1 & 1145.07 & 1 & 1.24 & 72985.50 & 1 & 1838.10 & 1 & 39.71 & 7472.57 & 1 & 1190.00 & 1 & 6.28 & 2515.52 & 0.90 & - \\
\hline$f_{28}$ & 2601.23 & 1 & 710.80 & 0.17 & 3.66 & 430.43 & 1 & 65824.00 & 0.10 & 0.01 & 22052.87 & 1 & 556.03 & 1 & 39.66 & 1297.70 & 1 & 3045.57 & 1 & 0.43 & 659.42 & 0.80 & - \\
\hline$f_{29}$ & 6604.80 & 1 & 964.80 & 0.83 & 6.85 & 7855.87 & 1 & 1139.24 & 0.70 & 6.90 & 27776.70 & 1 & 2774.08 & 0.83 & 10.01 & 15825.03 & 1 & 2308.63 & 0.90 & 6.85 & 1814.93 & 1 & 2222.08 \\
\hline$f_{30}$ & 1320.87 & 1 & 360.20 & 1 & 3.67 & - & 0 & - & 0 & - & 5365.53 & 1 & 614.43 & 1 & 8.73 & 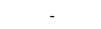 & 0 & - & 0 & - & 1062.87 & 1 & 858.47 \\
\hline$f_{31}$ & 1294.27 & 1 & 350.27 & 1 & 3.70 & & 0 & - & 0 & - & 5269.93 & 1 & 581.33 & 1 & 9.07 & & 0 & - & 0 & - & 1037.40 & 1 & 830.57 \\
\hline$f_{32}$ & 557.90 & 1 & 11.70 & 1 & 47.68 & 22.00 & 1 & 3.20 & 1 & 6.88 & 11576.67 & 1 & 20.77 & 1 & 557.46 & 448.87 & 1 & 7.97 & 1 & 56.34 & 35.53 & 1 & 8.57 \\
\hline$f_{33}$ & 2.20 & 1 & 2.17 & 1 & 1.02 & 2.03 & 1 & 1.77 & 1 & 1.15 & 2.53 & 1 & 2.30 & 1 & 1.10 & 1.93 & 1 & 1.77 & 1 & 1.09 & 3.23 & 1 & 2.03 \\
\hline$f_{34}$ & 73639.69 & 0.87 & 316.44 & 0.83 & 232.71 & 7668.17 & 0.97 & 194.38 & 0.87 & 39.45 & - & 0 & 506.76 & 0.83 & - & 43638.57 & 1 & 440.11 & 0.90 & 99.15 & 485.23 & 1 & 800.57 \\
\hline$f_{35}$ & - & 0 & - & 0 & - & & 0 & - & 0 & - & - & 0 & 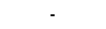 & 0 & - & & 0 & - & 0 & - & - & 0 & s \\
\hline$f_{36}$ & 30.73 & 1 & 24.17 & 1 & 1.27 & 24.50 & 1 & 25.97 & 1 & 0.94 & 26.57 & 1 & 25.23 & 1 & 1.05 & 19.90 & 1 & 24.73 & 1 & 0.80 & 32.30 & 1 & 25.33 \\
\hline$f_{37}$ & 39.10 & 1 & 9.23 & 1 & 4.23 & 37.63 & 1 & 6.47 & 1 & 5.82 & 54.63 & 1 & 20.27 & 1 & 2.70 & 34.33 & 1 & 12.13 & 1 & 2.83 & 15.63 & 1 & 8.93 \\
\hline & & & $\mathrm{DE} / \mathrm{rand} / 1$ & & & & & /best/1/exp & & & & $\mathrm{DE} / \mathrm{ran}$ & $\mathrm{d}$ to best $/ 1 / \mathrm{ex}$ & & & & $\mathrm{DE} / 1$ & rand/2/exp & & & & $\overline{\mathrm{DE} / \mathrm{t}}$ & best/2/exp \\
\hline$\stackrel{\breve{g}}{\Xi} \Xi$ & $\mathrm{DE}_{6}$ & & TSR & & & $\mathrm{DE}_{7}$ & & TSRVDE & & & $\mathrm{DE}_{8}$ & & TSRVDE $_{8}$ & & & $\mathrm{DE}_{9}$ & & TSRVDI & & & $\mathrm{DE}_{10}$ & & TSRVDE \\
\hline & $\mathrm{NFC}$ & S.R & $\mathrm{NFC}$ & S.R & A.R & NFC & S.R & NFC & S.R & A.R & NFC & S.R & $\mathrm{NFC}$ & S.R & A.R & NFC & S.R & $\mathrm{NFC}$ & S.R & A.R & NFC & S.R & $\mathrm{NFC}$ \\
\hline$f_{1}$ & 532.07 & 1 & 447.90 & 1 & 1.19 & 480.07 & 1 & 429.50 & 1 & 1.12 & 640.27 & 1 & 523.90 & 1 & 1.22 & 585.03 & 1 & 488.20 & 1 & 1.20 & 620.07 & 1 & 575.70 \\
\hline$f_{2}$ & 608.20 & 1 & 508.67 & 1 & 1.20 & 544.80 & 1 & 486.33 & 1 & 1.1 & 741.03 & 1 & 590.27 & 1 & 1.26 & 669.67 & 1 & 558.47 & 1 & 1.20 & 711.70 & 1 & 660.77 \\
\hline$f_{3}$ & 84116.63 & 1 & 58867.60 & 1 & 1.43 & 83516.64 & 0.47 & 86969.00 & 0.07 & 0.96 & 94412.13 & 1 & 58836.27 & 1 & 1.60 & 91624.93 & 1 & 62195.77 & 1 & 1.47 & 41334.90 & 1 & - \\
\hline$f_{4}$ & - & 0 & - & 0 & 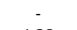 & & 0 & - & 0 & 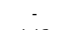 & 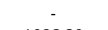 & 0 & & 0 & 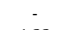 & & 0 & $\therefore$ & 0 & 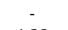 & 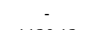 & 0 & $\therefore$ \\
\hline$f_{5}$ & 864.90 & 1 & 675.04 & 0.77 & 1.28 & 753.72 & 0.97 & 665.30 & 0.33 & 1.13 & 1098.80 & 1 & 824.97 & 1 & 1. & 977.40 & 1 & 762.40 & 1 & 1.28 & 1130.13 & 1 & 1013.73 \\
\hline$f_{6}$ & 1092.63 & 1 & 1777.63 & 1 & 0.61 & 800.74 & 0.90 & 680.92 & 0.87 & 1.1 & 1660.67 & 1 & 912.37 & 1 & 1.8 & 1288.00 & 1 & 755.79 & 0.93 & 1.70 & 1266.20 & 1 & 938.30 \\
\hline$f_{7}$ & 407.93 & 1 & 201.79 & 0.93 & 2.5 & 269.83 & 1 & 164.48 & 0.97 & 1. & 487.87 & 1 & 228.20 & 1 & 2.14 & 391.97 & 1 & 196.27 & 1 & 2.0 & 476.67 & 1 & 309.13 \\
\hline$f_{8}$ & 1054.57 & 1 & 865.27 & 0.5 & 1.22 & 910.40 & 1 & 825.25 & 0.27 & 1.1 & 1328.53 & 1 & 996.90 & 1 & 1.33 & 1180.80 & 1 & 936.93 & 1 & 1.2 & 1323.90 & 1 & 1238.07 \\
\hline$f_{g}$ & 291.70 & 1 & 253.23 & 1 & 1.15 & 261.20 & 1 & 236.90 & 1 & 1.10 & 340.97 & 1 & 289.47 & 1 & 1.18 & 318.37 & 1 & 270.27 & 1 & 1.18 & 323.57 & 1 & 305.63 \\
\hline$f_{10}$ & 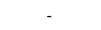 & 0 & . & 0 & & & 0 & - & 0 & 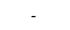 & & 0 & 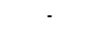 & 0 & & & 0 & . & 0 & 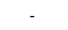 & & 0 & - \\
\hline$f_{11}$ & 996.57 & 1 & 813.96 & 0.93 & 1.22 & 873.90 & 1 & 763.14 & 0.93 & 1.1 & 1219.93 & 1 & 958.90 & 1 & 1.27 & 1104.67 & 1 & 905.47 & 1 & 1.22 & 1188.80 & 1 & 1084.43 \\
\hline$f_{12}$ & 368.93 & 1 & 315.20 & 1 & 1.17 & 332.93 & 1 & 303.23 & 1 & 1.10 & 445.70 & 1 & 366.70 & 1 & 1.22 & 401.80 & 1 & 343.50 & 1 & 1.17 & 426.67 & 1 & 397.37 \\
\hline$f_{13}$ & - & 0 & - & 0 & - & . & 0 & - & 0 & - & - & 0 & s & 0 & 列 & 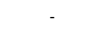 & 0 & 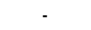 & 0 & - & $x_{1}+2$ & 0 & - \\
\hline$f_{14}$ & 275.30 & 1 & 239.33 & 1 & 1.15 & 254.60 & 1 & 230.83 & 1 & 1.10 & 331.63 & 1 & 274.30 & 1 & 1.21 & 300.57 & 1 & 262.43 & 1 & 1.15 & 312.67 & 1 & 299.37 \\
\hline$f_{15}$ & 4469.67 & 1 & 3677.60 & 1 & 1.22 & 4755.73 & 1 & 6520.60 & 1 & 0.73 & 4036.17 & 1 & 4342.40 & 1 & 0.93 & 2837.87 & 1 & 3769.80 & 1 & 0.75 & 3605.73 & 1 & 3361.90 \\
\hline$f_{16}$ & - & 0 & - & 0 & & & 0 & - & 0 & 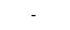 & & 0 & & 0 & & & 0 & - & 0 & - & & 0 & - \\
\hline$f_{17}$ & 7525.50 & 1 & 2979.20 & 0.50 & 2.5 & 4805.41 & 0.90 & 9324.75 & 0.13 & 0. & 17685.00 & 1 & 4423. & 1 & 4.0 & 10810.27 & 1 & 3955.24 & 0.97 & 2.73 & 13253.67 & 1 & 5821.03 \\
\hline$f_{18}$ & 96.03 & 1 & 85.60 & 1 & 1.12 & 86.30 & 1 & 80.73 & 1 & 1. & 113.97 & 1 & 104.93 & 1 & 1.09 & 104.90 & 1 & $\mathbf{9 4 . 8 7}$ & 1 & 1.11 & 135.93 & 1 & 140.00 \\
\hline$f_{19}$ & 454.00 & 1 & 374.03 & 1 & 1.21 & 405.37 & 1 & 361.37 & 1 & 1.12 & 560.17 & 1 & 440.60 & 1 & 1.27 & 504.83 & 1 & 412.80 & 1 & 1.22 & 554.93 & 1 & 505.60 \\
\hline$f_{20}$ & 153416.62 & 0.87 & 4313.37 & 1 & 35.57 & 124603.00 & 0.60 & 6760.27 & 1 & 18.43 & 218312.60 & 0.67 & 5965.83 & 1 & 36.59 & 166125.74 & 0.63 & 3635.73 & 1 & 45.69 & 89996.13 & 1 & 1687.00 \\
\hline$f_{21}$ & - & 0 & - & 0 & - & - & 0 & . & 0 & - & - & 0 & . & 0 & res & & 0 & 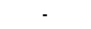 & 0 & - & s & 0 & - \\
\hline$f_{22}$ & 533.27 & 1 & 439.7 & 0.97 & 1.21 & 471.7 & 1 & 418.6 & 1 & 1.1 & 651.7 & 1 & 516.3 & 1 & 1.26 & 585.3 & 1 & 483.5 & 1 & 1.21 & 719.43 & 1 & 652.57 \\
\hline$f_{23}$ & 926.77 & 1 & 779.57 & 1 & 1. & 830.30 & 1 & 740.31 & 0.97 & 1.12 & 1135.13 & 1 & 906.93 & 1 & 1.2 & 1023.07 & 1 & 849.67 & 1 & 1. & 1099.77 & 1 & 1014.67 \\
\hline$f_{24}$ & 526.07 & 1 & 446.03 & 1 & 1. & 472.07 & 1 & 428.37 & 1 & 1.10 & 637.43 & 1 & 509.83 & 1 & 1.25 & 578.50 & 1 & 481.67 & 1 & 1.20 & 609.13 & 1 & 564.33 \\
\hline$f_{25}$ & 34088.08 & 0.43 & 3393.00 & 0.03 & 10.0 & 8505.00 & 0.13 & - & 0 & - & 127052.50 & 0.13 & 17107.00 & 0.23 & 7.43 & 45470.50 & 0.07 & 72285.33 & 0.10 & 0.63 & 42413.42 & 0.80 & 139249.00 \\
\hline$f_{26}$ & 714.63 & 1 & 600.57 & 1 & 1.19 & 639.50 & 1 & 577.28 & 0.97 & 1.11 & 872.83 & 1 & 702.47 & 1 & 1.24 & 787.60 & 1 & 659.63 & 1 & 1.19 & 843.90 & 1 & 780.30 \\
\hline$f_{27}$ & 738.83 & 1 & 610.17 & 1 & 1.21 & 661.0 & 1 & 583.66 & 0.97 & 1.13 & 901.87 & 1 & 710.23 & 1 & 1.2 & 827. & 1 & 665.03 & 1 & 1.24 & 876.97 & 1 & 805.13 \\
\hline$f_{28}$ & 5034.27 & 1 & - & 0 & - & 4555.37 & 1 & & 0 & - & 3849.23 & 1 & 3235.63 & 1 & 1.1 & 6003.00 & 1 & 2449.00 & 1 & 2.45 & & 0 & 1990.33 \\
\hline$f_{29}$ & 200.93 & 1 & 187.60 & 1 & 1.07 & 205.93 & 1 & 175.97 & 1 & 1.17 & 260.60 & 1 & 202.87 & 1 & 1.28 & 231.30 & 1 & 203.40 & 1 & 1.14 & 171.63 & 1 & 160.37 \\
\hline$f_{30}$ & 796.70 & 1 & 705.10 & 0.97 & 1.13 & 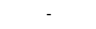 & 0 & 711.53 & 0.57 & 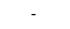 & 940.10 & 1 & 768.23 & 1 & 1.2 & 841.30 & 1 & 1062.57 & 1 & 0.79 & 1397.10 & 1 & .70 \\
\hline$f_{31}$ & 795.30 & 1 & 705.13 & 1 & 1.13 & - & 0 & 640.67 & 1 & - & 939.03 & 1 & 782.03 & 1 & 1.20 & 844.73 & 1 & 736.80 & 1 & 1.15 & 1401.60 & 1 & 1375.90 \\
\hline$f_{32}$ & 80.37 & 1 & 65.70 & 1 & 1.22 & 72.03 & 1 & 62.80 & 1 & 1.15 & 89.33 & 1 & 79.53 & 1 & 1.12 & 86.03 & 1 & 74.37 & 1 & 1.16 & 77.87 & 1 & 74.10 \\
\hline
\end{tabular}




\begin{tabular}{|c|c|c|c|c|c|c|c|c|c|c|c|c|c|c|c|c|c|c|c|c|c|c|c|c|c|}
\hline$f_{33}$ & 2.43 & 1 & 2.43 & 1 & 1.00 & 2.17 & 1 & 2.57 & 1 & 0.84 & 2.47 & 1 & 2.33 & 1 & 1.06 & 2.63 & 1 & 2.23 & 1 & 1.18 & 2.57 & 1 & 2.20 & 1 & 1.17 \\
\hline$f_{34}$ & 1601.77 & 1 & 1068.61 & 0.60 & 1.50 & 1155.40 & 1 & 954.93 & 0.47 & 1.21 & 2530.23 & 1 & 1440.90 & 1 & 1.76 & 1776.30 & 1 & 1212.57 & 1 & 1.46 & 2499.97 & 1 & 2039.37 & 1 & 1.23 \\
\hline$f_{35}$ & - & 0 & 5165.77 & 1 & 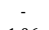 & - & 0 & - & 0 & $\therefore$ & - & 0 & 25698.17 & 1 & - & 16453.44 & 0.30 & 24783.50 & 0.07 & 0.66 & 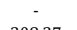 & 0 & - & 0 & - \\
\hline$f_{36}$ & 292.37 & 1 & 275.87 & 1 & 1.06 & 270.80 & 1 & 237.85 & 0.87 & 1.14 & 379.63 & 1 & 268.50 & 1 & 1.41 & 316.10 & 1 & 274.30 & 1 & 1.15 & 308.37 & 1 & 264.90 & 1 & 1.16 \\
\hline 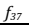 & 16.50 & 1 & 15.23 & 1 & 1.08 & 17.50 & 1 & 16.77 & 1 & 1.04 & 16.77 & 1 & 18.53 & 1 & 0.90 & 17.17 & 1 & 15.77 & 1 & 1.09 & 15.63 & 1 & 15.77 & , & 0.99 \\
\hline
\end{tabular}

Table 6: Summary of results Number of Functions Calls (NFC) best, Average Success Rate (A.S.R) and Average Acceleration Rate (A.A.R)

\begin{tabular}{|c|c|c|c|c|c|c|c|c|c|c|c|c|c|c|c|c|c|}
\hline \multicolumn{6}{|c|}{ 10D } & \multicolumn{6}{|c|}{$20 \mathrm{D}$} & \multicolumn{6}{|c|}{$30 \mathrm{D}$} \\
\hline Variant & Best & A.S.R & Variant & Best & A.S.R & Variant & Best & A.S.R & Variant & Best & A.S.R & Variant & Best & A.S.R & Variant & Best & A.S.R \\
\hline $\mathrm{DE}_{1}$ & 1 & 0.78 & $\mathrm{DE}_{6}$ & 2 & 0.81 & $\mathrm{DE}_{1}$ & 2 & 0.72 & $\mathrm{DE}_{6}$ & 4 & 0.81 & $\mathrm{DE}_{1}$ & 1 & 0.71 & $\mathrm{DE}_{6}$ & 3 & 0.80 \\
\hline TSRVDE $_{1}$ & 30 & 0.70 & $\mathrm{TSRVDE}_{6}$ & 29 & 0.73 & TSRVDE $_{1}$ & 31 & 0.72 & TSRVDE $_{6}$ & 28 & 0.73 & TSRVDE $_{1}$ & 32 & 0.73 & TSRVDE $_{6}$ & 30 & 0.74 \\
\hline A.A.R & \multicolumn{2}{|c|}{23.50} & A.A.R & \multicolumn{2}{|c|}{1.66} & A.A.R & \multicolumn{2}{|c|}{49.37} & A.A.R & \multicolumn{2}{|c|}{2.02} & A.A.R & \multicolumn{2}{|c|}{95.58} & A.A.R & \multicolumn{2}{|c|}{2.68} \\
\hline $\mathrm{DE}_{2}$ & 7 & 0.76 & $\mathrm{DE}_{7}$ & 3 & 0.77 & $\mathrm{DE}_{2}$ & 5 & 0.79 & $\mathrm{DE}_{7}$ & 8 & 0.76 & $\mathrm{DE}_{2}$ & 8 & 0.75 & $\mathrm{DE}_{7}$ & 6 & 0.71 \\
\hline A.A.R & \multicolumn{2}{|c|}{4.27} & A.A.R & \multicolumn{2}{|c|}{1.29} & A.A.R & \multicolumn{2}{|c|}{19.41} & A.A.R & \multicolumn{2}{|c|}{1.90} & A.A.R & \multicolumn{2}{|c|}{27.64} & A.A.R & \multicolumn{2}{|c|}{1.73} \\
\hline $\mathrm{DE}_{3}$ & 3 & 0.72 & $\mathrm{DE}_{8}$ & 2 & 0.82 & $\mathrm{DE}_{3}$ & 0 & 0.67 & $\mathrm{DE}_{8}$ & 1 & 0.77 & $\mathrm{DE}_{3}$ & 1 & 0.68 & $\mathrm{DE}_{8}$ & 2 & 0.78 \\
\hline TSRVDE $_{3}$ & 29 & 0.72 & TSRVDE8 & 31 & 0.79 & TSRVDE $_{3}$ & 33 & 0.75 & TSRVDE8 & 31 & 0.80 & TSRVDE $_{3}$ & 33 & 0.78 & TSRVDE$_{8}$ & 30 & 0.82 \\
\hline A.A.R & \multicolumn{2}{|c|}{29.10} & A.A.R & \multicolumn{2}{|c|}{2.54} & A.A.R & \multicolumn{2}{|c|}{43.05} & A.A.R & \multicolumn{2}{|c|}{2.88} & A.A.R & \multicolumn{2}{|c|}{113.07} & A.A.R & \multicolumn{2}{|c|}{2.72} \\
\hline $\mathrm{DE}_{4}$ & 5 & 0.78 & $\mathrm{DE}_{9}$ & 5 & 0.81 & $\mathrm{DE}_{4}$ & 2 & 0.70 & $\mathrm{DE}_{9}$ & 2 & 0.78 & $\mathrm{DE}_{4}$ & 1 & 0.65 & $\mathrm{DE}_{9}$ & 4 & 0.79 \\
\hline A.A.R & \multicolumn{2}{|c|}{39.44} & A.A.R & \multicolumn{2}{|c|}{1.65} & A.A.R & \multicolumn{2}{|c|}{14.59} & A.A.R & \multicolumn{2}{|c|}{2.16} & A.A.R & \multicolumn{2}{|c|}{19.82} & A.A.R & \multicolumn{2}{|c|}{2.66} \\
\hline $\mathrm{DE}_{5}$ & 7 & 0.75 & $\mathrm{DE}_{10}$ & 4 & 0.77 & $\mathrm{DE}_{5}$ & 13 & 0.73 & $\mathrm{DE}_{10}$ & 4 & 0.76 & $\mathrm{DE}_{5}$ & 13 & 0.72 & $\mathrm{DE}_{10}$ & 4 & 0.78 \\
\hline TSRVDE $_{5}$ & 23 & 0.65 & $\operatorname{TSRVDE}_{10}$ & 27 & 0.76 & TSRVDE $_{5}$ & 20 & 0.63 & TSRVDE $_{10}$ & 27 & 0.73 & TSRVDE $_{5}$ & 20 & 0.66 & $\operatorname{TSRVDE}_{10}$ & 27 & 0.74 \\
\hline A.A.R & \multicolumn{2}{|c|}{2.97} & A.A.R & \multicolumn{2}{|c|}{1.28} & A.A.R & \multicolumn{2}{|c|}{3.12} & A.A.R & \multicolumn{2}{|c|}{2.33} & A.A.R & \multicolumn{2}{|c|}{4.04} & A.A.R & & \\
\hline
\end{tabular}

From research results it can be summarized that TSRVDE mutation strategies have better performance than DE mutation strategies in most of the cases of NFC performance parameter while in few cases TSRVDE mutation strategies have worse performance than DE mutation strategies for $10 \mathrm{D}$, 20D and 30D respectively. The convergence speed of DE and TSRVDE mutation strategies can be observed in acceleration rate and average acceleration result that shows TSRVDE strategies have fast convergence than DE mutation strategies in almost all cases. Success rate and average success rate result shows that in some cases DE and TRSRVE mutation strategies have about similar performance while TSRVDE mutation strategies are slightly better in most of the cases.

So it can be observed that the selection of parent in generating $\mathrm{DE}$ trial vector have significant affect on the performance of $\mathrm{DE}$ algorithm. The vectors used in trial vector generation that should be selected by some criteria can be expected to produce better results than selecting them by random techniques. The tournament of small size $(\mathrm{k}=3)$ is used in proposed TSDE that escapes the selection of most worst performing individuals of population.

Fig. 2 and Fig. 3 contain the convergence graphs of average of best values taken at some specific generations during the population evolution. Each sub-graph in the convergence graph shows the DE

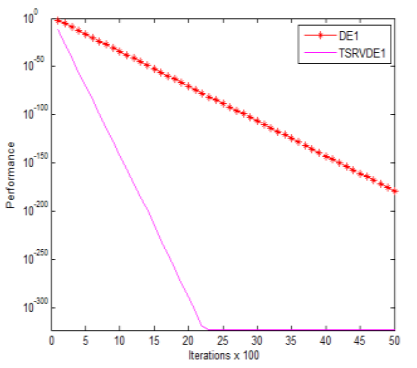

$\mathrm{a}_{1}$

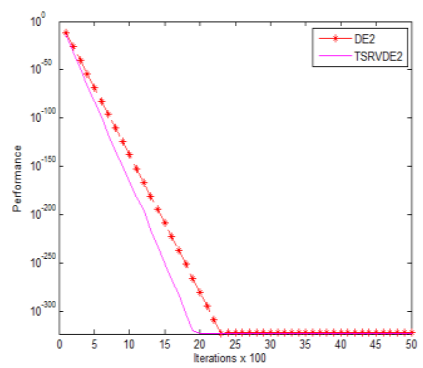

$\mathrm{b}_{1}$ mutation strategy and its corresponding TSRVDE mutation that shows performance along y-axis and iterations against $\mathrm{x}$-axis as a multiple of 100 . Convergence graphs of some selected functions are given in the paper for 10 dimensions only using 10,000 generation for all functions and all mutation strategies of DE and TSRVDE. Convergence graphs of $\mathrm{DE}$ mutation strategies ( $\left.\mathrm{DE}_{1} \ldots . . \mathrm{DE}_{10}\right)$ and TSRVDE mutation strategies (TSRVDE 1 ...... TSRVDE 10 ) are generation for functions $-f_{10}\left(a_{1}-j_{1}\right), f_{13}\left(a_{2}-j_{2}\right), f_{16}\left(a_{3}-\right.$ $\left.\mathrm{j}_{3}\right), \mathrm{f}_{19}\left(\mathrm{a}_{4}-\mathrm{j}_{4}\right)$. It can be observed from convergence graphs that TSRVDE mutation strategies performance better than DE mutation strategies in most of the cases and in few cased DE mutation strategies performance is better.

\section{Conclusion and future work}

In DE algorithm, trial vector generation strategies have a significant influence in generating offspring population. Various vectors like current, best and random are used to form the equation of DE trial vector. Selection of worst performing random vector(s) from the current population should be reduced to speed up the convergence speed of $\mathrm{DE}$ algorithm. In this paper a novel framework in DE is employed that utilizes tournament selection mechanism in selecting random vector.

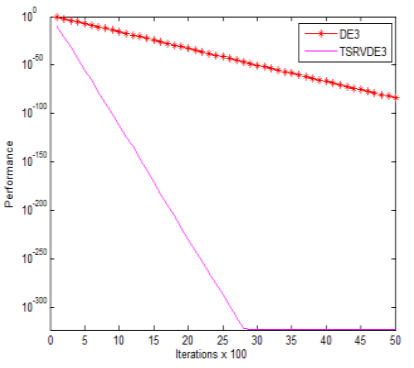

$\mathrm{C}_{1}$

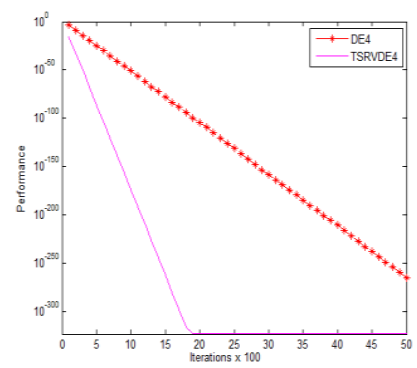

$\mathrm{d}_{1}$ 


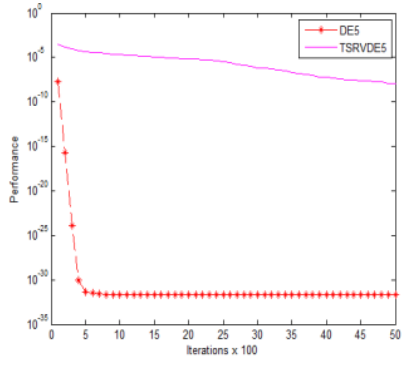

$\mathrm{e}_{1}$

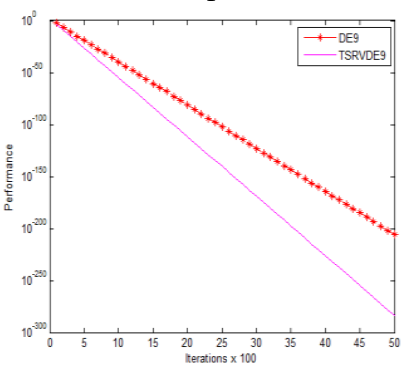

$\mathrm{i}_{1}$

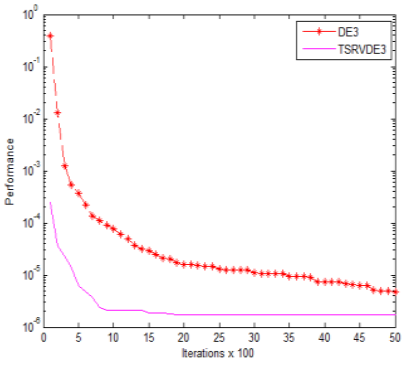

$\mathrm{C}_{2}$

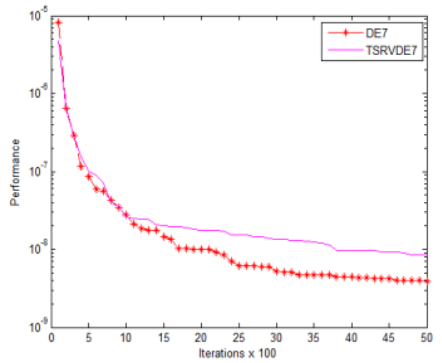

$\mathrm{g}_{2}$

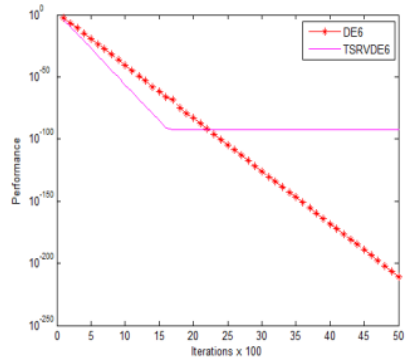

$\mathrm{f}_{1}$

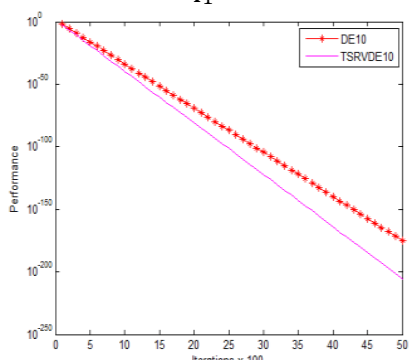

$\mathrm{j}_{1}$

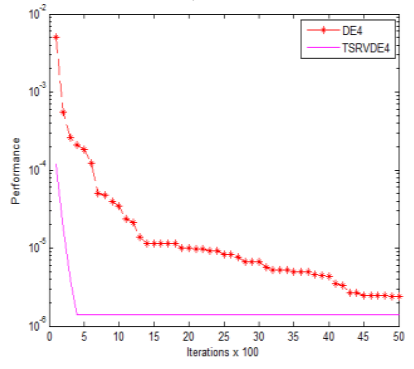

$\mathrm{d}_{2}$

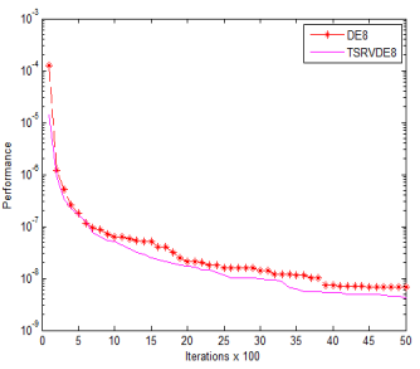

$\mathrm{h}_{2}$
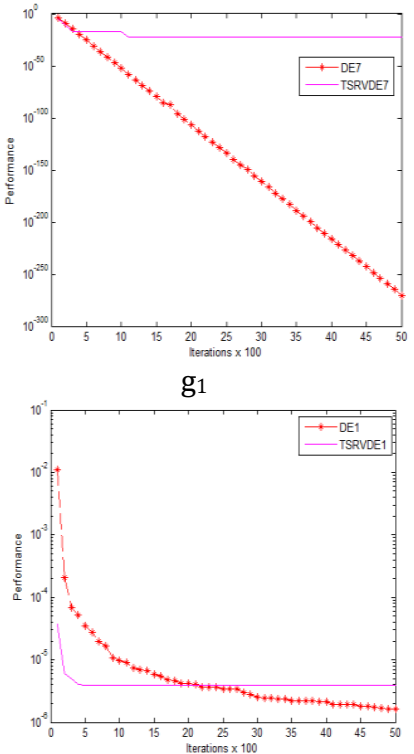

a2

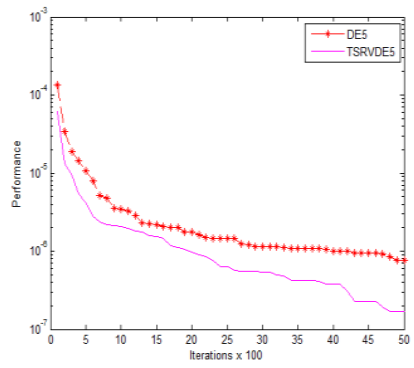

$\mathrm{e}_{2}$

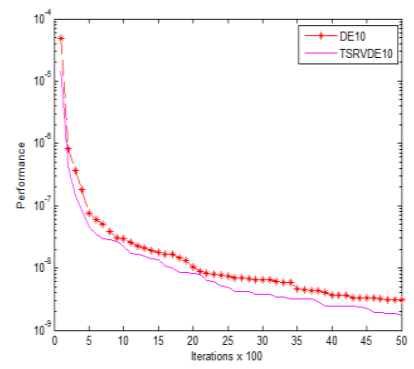

$\mathrm{i}_{2}$

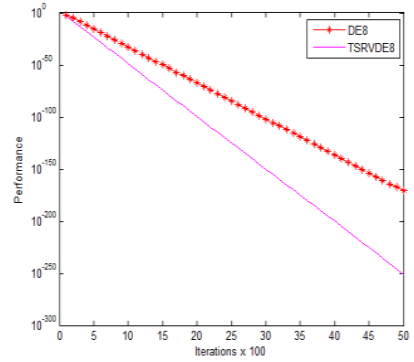

$\mathrm{h}_{1}$

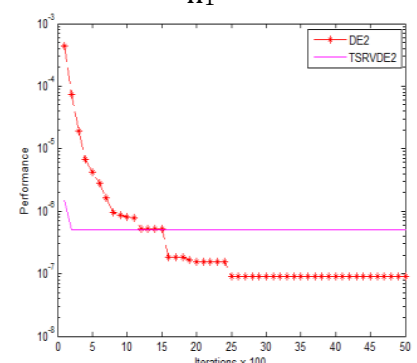

$\mathrm{b}_{2}$

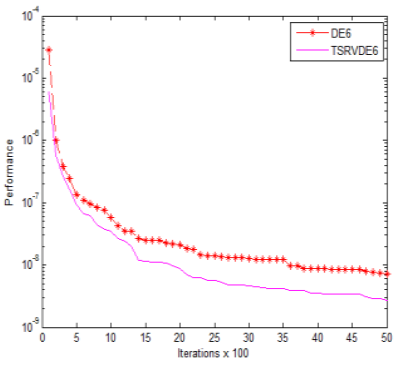

$\mathrm{f}_{2}$

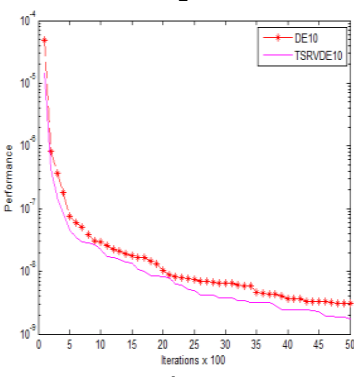

$\mathrm{j} 2$

Fig. 2: DE and TSRVDE variants 10D Convergence graphs for function $\mathrm{f}_{10}\left(\mathrm{a}_{1}-\mathrm{j}_{1}\right), \mathrm{f}_{13}\left(\mathrm{a}_{2}-\mathrm{j}_{2}\right)$

TSRVDE approach select best performing individual from a tournament of small size $(\mathrm{k}=3)$ by using tournament selection. A comprehensive set of commonly used multimodal global optimization function problems given in the appendix section is used to access the performance of TSRVDE. Corresponding to most commonly used mutation strategies of DE algorithm given in Table 1, TSRVDE mutation strategies given in Table 2 are considered in the experimental results. Well-known

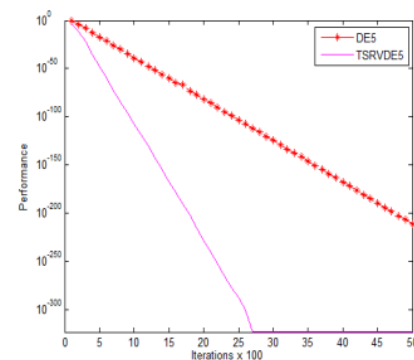

a3

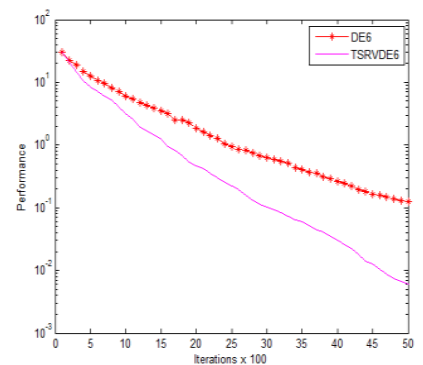

$\mathrm{b}_{3}$

performance parameters NFC, AR and SR are used in comparing the performance of DE and TSRVDE mutation strategies. From experimental results it can be observed that TSRVDE has significantly better convergence speed than DE mutation strategies. In almost all cases of Number of Function Calls of TSRVDE mutation strategies outperforms DE mutation strategies. Similarly TSRVDE mutation strategies perform better than DE in almost all cases of Acceleration Rate parameter.

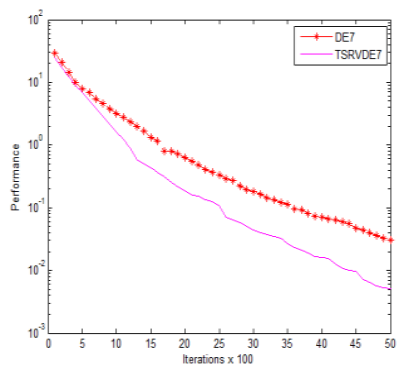

c3

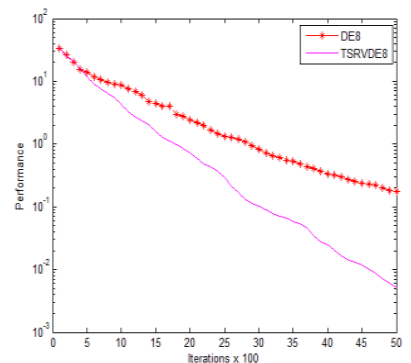

$\mathrm{d}_{3}$ 


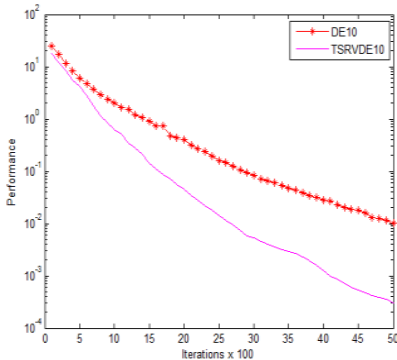

$\mathrm{e}_{3}$

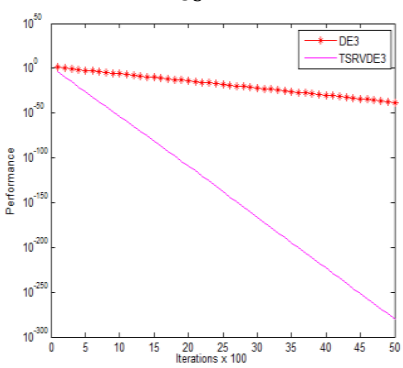

i3

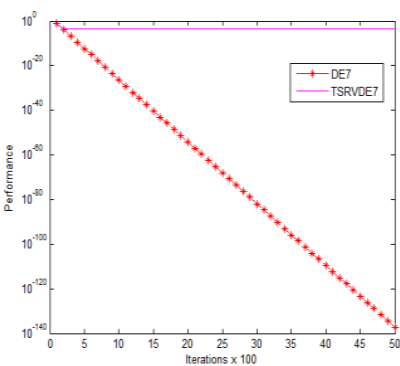

$\mathrm{C}_{4}$

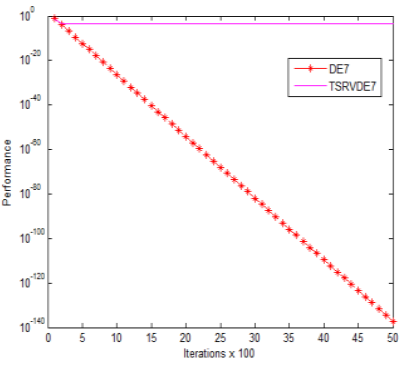

$\mathrm{g}_{4}$

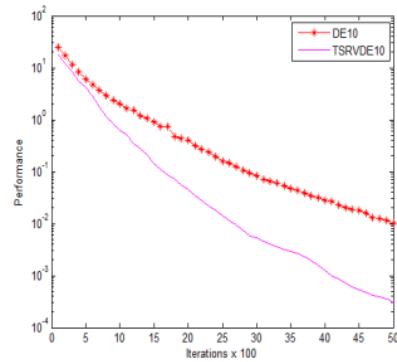

$\mathrm{f}_{3}$

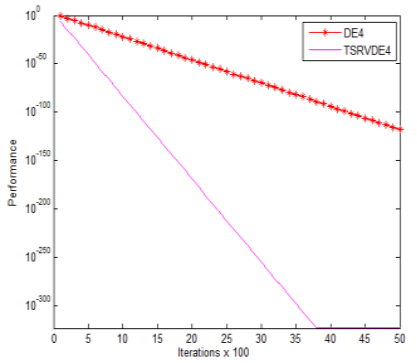

j3

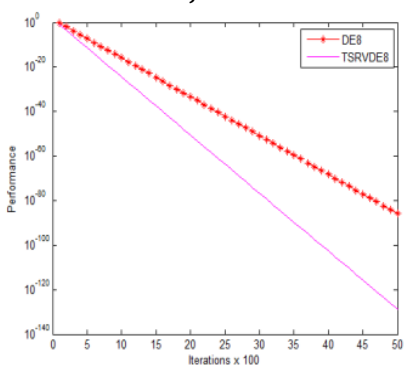

$\mathrm{d}_{4}$

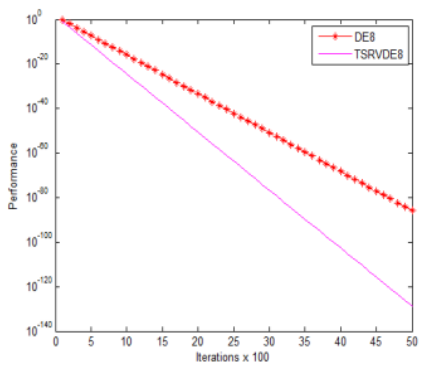

$\mathrm{h}_{4}$

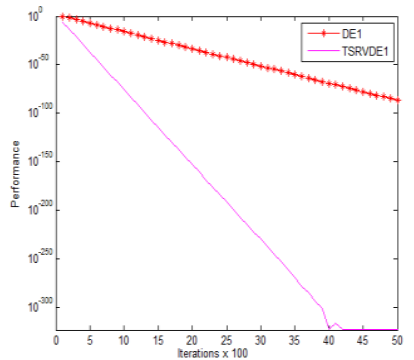

$\mathrm{g}_{3}$

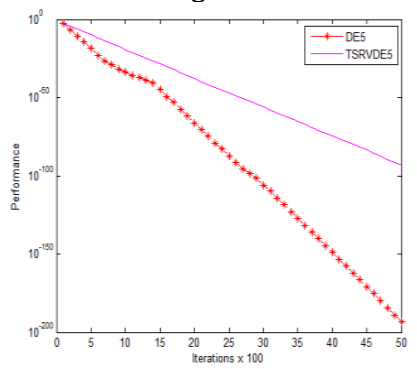

$a_{4}$

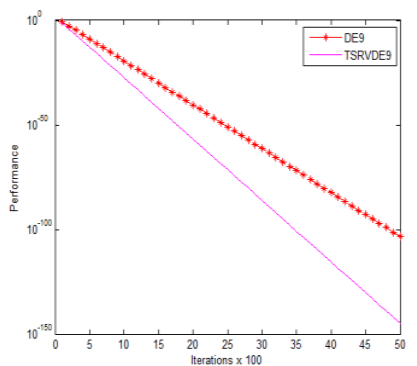

$\mathrm{e}_{4}$

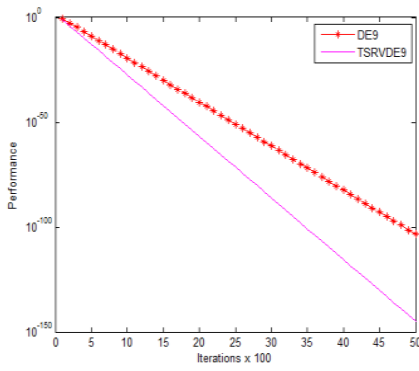

i4

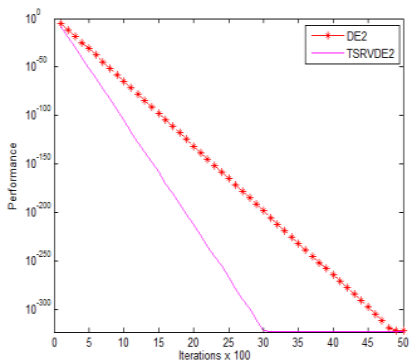

$\mathrm{h}_{3}$

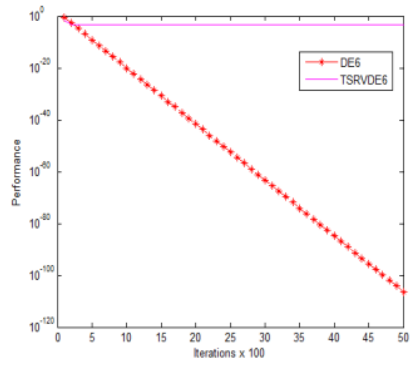

$\mathrm{b}_{4}$

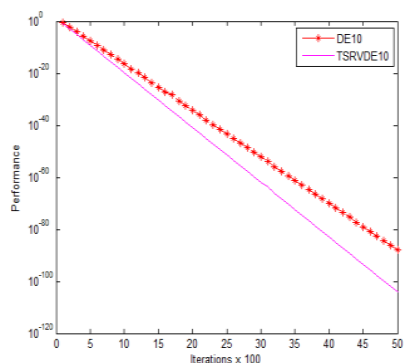

$\mathrm{f}_{4}$

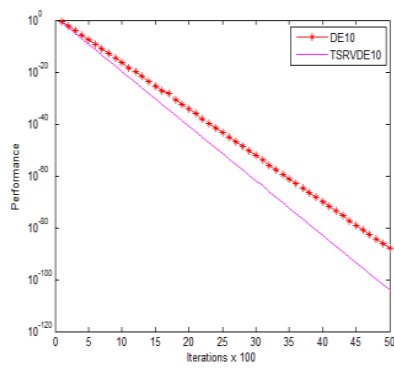

j4

Fig. 3: DE and TSRVDE 10D Convergence graphs for function $\mathrm{f}_{16}\left(\mathrm{a}_{3}-\mathrm{j}_{3}\right), \mathrm{f}_{19}\left(\mathrm{a}_{4}-\mathrm{j}_{4}\right)$

TSRVDE mutation strategies have either comparable or slightly less success rate than DE mutation strategies. An effort is made to introduce some new direction in DE research that will prove to be a significant addition in DE algorithm. The depth insight of TSRVDE parameter adaptation and its exploration in other research dimensions can be future challenge of this research work.

\section{References}

Ali M, Pant M, Abraham A, and Snasel V (2011). Differential evolution using mixed strategies in competitive environment. International Journal of Innovative Computing, Information and Control, 7(8): 5063-5084.

Ali M, Pant M, and Abraham A (2009a). Simplex differential evolution. Acta Polytechnica Hungarica, 6(5): 95-115.

Ali M, Pant M, and Singh VP (2009b). An improved differential evolution algorithm for real parameter optimization problems. International Journal of Recent Trends in Engineering, 1(5): 63-65.
Brest J, Greiner S, Boskovic B, Mernik M, and Zumer V (2006). Selfadapting control parameters in differential evolution: A comparative study on numerical benchmark problems. IEEE Transactions on Evolutionary Computation, 10(6): 646-657.

Cai Y and Wang J (2013). Differential evolution with neighborhood and direction information for numerical optimization. IEEE Transactions on Cybernetics, 43(6): 2202-2215.

Chiou JP, Chang CF, and Su CT (2005). Variable scaling hybrid differential evolution for solving network reconfiguration of distribution systems. IEEE Transactions on Power Systems, 20(2): 668-674.

Choi TJ, Ahn CW, and An J (2013). An adaptive cauchy differential evolution algorithm for global numerical optimization. The Scientific World Journal, 2013: Article ID 969734, 12 pages. https:// doi.org/10.1155/2013/969734

Das S, Abraham A, and Konar A (2008b). Particle swarm optimization and differential evolution algorithms: Technical analysis, applications and hybridization perspectives. In: Liu Y, Sun A, Loh HT, Lu WF, and Lim EP (Eds.), Advances of Computational Intelligence in Industrial Systems: 1-38. Springer Berlin Heidelberg, Heidelberg Germany.

Das S, Abraham A, Chakraborty UK, and Konar A (2009). Differential evolution using a neighborhood-based mutation 
operator. IEEE Transactions on Evolutionary Computation, 13(3): 526-553.

Das S, Dasgupta S, Biswas A, and Abraham A (2008a). Automatic circle detection on images with annealed differential evolution. In the $8^{\text {th }}$ International Conference on Hybrid Intelligent Systems, IEEE, Barcelona, Spain: 684-689. https://doi.org/10.1109/HIS.2008.169

De Oliveira GTS and Saramago SFP (2008). A contribution to the study about differential evolution. Ciência \& Engenharia, 16(1/2): 1-8.

Dos Santos Coelho L and Guerra FA (2008). B-spline neural network design using improved differential evolution for identification of an experimental nonlinear process. Applied Soft Computing, 8(4): 1513-1522.

Elsayed SM, Sarker RA, and Essam DL (2013). An improved selfadaptive differential evolution algorithm for optimization problems. IEEE Transactions on Industrial Informatics, 9(1): 89-99.

Epitropakis MG, Tasoulis DK, Pavlidis NG, Plagianakos VP, and Vrahatis MN (2011). Enhancing differential evolution utilizing proximity-based mutation operators. IEEE Transactions on Evolutionary Computation, 15(1): 99-119.

Garlapati VK and Banerji R (2010). Optimization of lipas production using differential evoltuion. Biotechnology and Bipprocess engineering, 15(2): 254-260.

Ghosh A, Das S, Chowdhury A, and Giri R (2011). An improved differential evolution algorithm with fitness-based adaptation of the control parameters. Information Sciences, 181(18): 3749-3765.

Gong W, Cai Z, Ling CX, and Li H (2011). Enhanced differential evolution with adaptive strategies for numerical optimization. IEEE Transactions on Systems, Man, and Cybernetics, Part B (Cybernetics), 41(2): 397-413.

Guo JL and Li JY (2009). Pattern synthesis of conformal array antenna in the presence of platform using differential evolution algorithm. IEEE Transactions on Antennas and Propagation, 57(9): 2615-2621.

Halder U, Das S, and Maity D (2013). A cluster-based differential evolution algorithm with external archive for optimization in dynamic environments. IEEE Transactions on Cybernetics, 43(3): 881-887.

Islam SM, Das S, Ghosh S, Roy S, and Suganthan PN (2012). An adaptive differential evolution algorithm with novel mutation and crossover strategies for global numerical optimization. IEEE Transactions on Systems, Man, and Cybernetics, Part B (Cybernetics), 42(2): 482-500.

Kang L, Wu L, Wei Y, Yang B, and Song H (2014). A highly accurate dense approach for homography estimation using modified differential evolution. Engineering Applications of Artificial Intelligence, 31: 68-77.

Li X, Li WT, Shi XW, Yang J, and Yu JF (2013). Modified differential evolution algorithm for pattern synthesis of antenna arrays. Progress in Electromagnetics Research, 137: 371-388.

Liang W, Zhang L, and Wang M (2011). The chaos differential evolution optimization algorithm and its application to support vector regression machine. Journal of Software, 6(7): 1297-1304.

Liu J, and Lampinen J (2005). A fuzzy adaptive differential evolution algorithm. Soft Computing, 9: 448-462.

Liu Y, Tang X, Tao G, and Joshi SM (2006). Adaptive failure compensation for aircraft tracking control using engine differential based model. In the American Control Conference, IEEE, Minneapolis, USA: 5984-5989. https://doi.org/10.1109/ACC.2006.1657680

Luitel B and Venayagamoorthy GK (2008). Differential evolution particle swarm optimization for digital filter design. In the IEEE Congress on Evolutionary Computation (CEC '08), IEEE
World Congress on Computational Intelligence, IEEE, Hong Kong, $\quad$ China: $3954 \quad$ - 3961. https://doi.org/10.1109/CEC.2008.4631335

Mallipeddi R, Suganthan PN, Pan QK, and Tasgetiren MF (2011). Differential evolution algorithm with ensemble of parameters and mutation strategies. Applied Soft Computing, 11(2): 16791696.

Mezura-Montes E, Reyes JV, and Coello Coello CA (2006). A comparative study of differential evolution variants for global optimization. In the 8th annual conference on Genetic and evolutionary computation (GECCO '06), ACM, Washington, USA: 485-492. https://doi.org/10.1145/1143997.1144086

Mininno E, Neri F, Cupertino F, and Naso D (2011). Compact differential evolution. IEEE Transactions on Evolutionary Computation, 15(1): 32-54.

Piotrowski AP, and Napiorkowski JJ (2010). The grouping differential evolution algorithm for multi-dimensional optimization problems. Control and Cybernetics, 39(2): 527550.

Price KV, Storn RM, and Lampinen JA (2005). The differential evolution algorithm. In: Price KV, Storn RM, and Lampinen JA (Eds.), Differential Evolution: A Practical Approach to Global Optimization: 37-134. Springer Berlin Heidelberg, Heidelberg Germany.

Qin AK, Huang VL, and Suganthan PN (2009). Differential evolution algorithm with strategy adaptation for global numerical optimization. IEEE Transactions on Evolutionary Computation, 2(13): 398-417.

Rahnamayan S, Tizhoosh HR, and Salama MM (2008). Oppositionbased differential evolution. IEEE transactions on evolutionary computation, 12(1): 64-79.

Secmen M and Tasgetiren MF (2013). Ensemble of differential evolution algorithms for electromagnetic target recognition problem. IET Radar, Sonar and Navigation, 7(7): 780-788.

Slowik A (2011). Application of an adaptive differential evolution algorithm with multiple trial vectors to artificial neural network training. IEEE Transactions On Industrial Electronics, 58(8): 3160-3167.

Storn R and Price K (1997). Differential evolution-A simple and efficient heuristic for global optimization over continuous spaces. Journal of Global Optimization, 11(4): 341-359.

Wang H, Rahnamayan S, Sun H, and Omran MG (2013). Gaussian bare-bones differential evolution. IEEE Transactions on Cybernetics, 43(2): 634-647.

Wang H, Wu Z, and Rahnamayan S (2010). Differential Evolution enhanced by neighborhood search. In the IEEE Congress on Evolutionary Computation, IEEE, Barcelona, Spain: 1-8. https://doi.org/10.1109/CEC.2010.5586418

Wang Y, Cai Z, and Zhang Q (2011). Differential evolution with composite trial vector generation strategies and control parameters. IEEE Transactions on Evolutionary Computation, 15(1): 55-66.

Wang Y, Cai Z, and Zhang Q (2012). Enhancing the search ability of differential evolution through orthogonal crossover. Information Sciences, 185: 153-177.

$\mathrm{Xu} \mathrm{X}$ and Li Y (2007). Comparison between particle swarm optimization, differential evolution and multi-parents crossover. In the International Conference on Computational Intelligence and Security, IEEE, Harbin, China: 124-127. https://doi.org/10.1109/CIS.2007.37

Xue-Feng Y, Yu J, and Qian F (2006). Adaptive mutation differential evolution algorithm and its application to estimate soft sensorparameters. Kongzhi Lilun yu Yingyong/ Control Theory and Applications, 23(5): 744-748.

Zaharie D (2003). Control of population diversity and adaptation in differential evolution algorithms. In the 9 th International Conference on Soft Computing, Brno, Czech Republic: 41-46. 
Zhang J and Sanderson AC (2009). JADE: adaptive differential evolution with optional external archive. IEEE Transactions on evolutionary computation, 13(5): 945-958.

Zhong JH, Shen M, Zhang J, Chung HSH, Shi YH, and Li Y (2013). A differential evolution algorithm with dual populations for solving periodic railway timetable scheduling problem. IEEE Transactions on Evolutionary Computation, 17(4): 512-527.

Zhou Y, Li X, and Gao L (2013). A differential evolution algorithm with intersect mutation operator. Applied Soft Computing, 13: 390-401. 